\title{
Safety and Quality Recommendations in Allergy Medicine (Spanish acronym, RESCAL)
}

\author{
Vidal $\mathrm{C}^{1}$, Antolín $\mathrm{D}^{2}$, Reaño $\mathrm{M}^{3}$, Valero $\mathrm{A}^{4}$, Sastre $\mathrm{J}^{5}$ \\ 'Vice President, SEAIC. Head of the Allergy Department, Complejo Hospitalario Universitario de Santiago. Adjunct Professor of Medicine. \\ Faculty of Medicine. University of Santiago de Compostela. \\ ${ }^{2}$ Secretary, SEAIC. Staff specialist, Department of Immune System Diseases and Allergy. Hospital Universitario Príncipe de Asturias. Department \\ of Medicine and Medical Specialties. Universidad de Alcalá. Alcalá de Henares. \\ ${ }^{3}$ President, SEAIC Quality Committee. Specialist physician, Allergy Unit. Hospital Puerta de Hierro, Majadahonda. Madrid. \\ ${ }^{4}$ President-elect, SEAIC. Allergy Section, Pulmonology and Allergy Department. Hospital Clínic de Barcelona. \\ 5President, SEAIC. Head of the Allergy Department, Fundación Jiménez Díaz, Madrid. Universidad Autónoma de Madrid.
}

Collaborators

Committee on Hymenoptera Allergy:

Dr. Teresa Alfaya and Dr. Berta Ruíz León

Asthma Committee:

Dr. Julio Delgado and Dr. Carmen Pérez Francés

Committee on Pediatric Allergy:

Dr. Carmelo Escudero and Dr. Paloma Ibañez

Committee on Cutaneous Allergy:

Dr. Lys Herráez and Dr. José Luís García Abujeta

Committee on Nursing Care in Allergy:

Ms. Cristina Mañas and Mr. Valentín López Carrasco

Immunotherapy Committee:

Dr. Ana I. Tabar and Dr. Eloina González Mancebo

Committee on Drug Allergy:

Dr. Pilar Berges, Dr. Marta López San Martín

and Dr. María José Torres

\section{SEAIC Board of Directors}

Dr. Javier Montoro Lacomba

(Vice Secretary-Treasurer for the SEAIC)

Dr. Maria José Álvarez Puebla

(board member)

Dr. Juan Fraj Lázaro

(board member)

Dr. Nancy R. Ortega Rodríguez

(board member)

\author{
Committee on Food Allergy: \\ Dr. Teresa Valbuena, Dr. Blanca García Figueroa \\ and Dr. Joan Bartra \\ Rhinoconjunctivitis Committee: \\ Dr. Francisco Vega and Dr. Teresa Dordal \\ Immunology Committee: \\ Dr. Moisés Labrador and Dr. Mariona Pascal \\ Aerobiology Committee: \\ Dr. Ángel Moral and Dr. Sergio Porcel \\ Quality Committee: \\ Dr. Francisco Javier Ruano, Dr. Jorge Darío Méndez, \\ Dr. Alfredo Iglesias and Dr. María Isabel Alvarado
}

Commission on Professional Issues:

Dr. Dolores Hernández

J Investig Allergol Clin Immunol 2018; Vol. 28, Suppl. 1: 1-39

\author{
Dr. Juan Carlos Miralles López \\ (board member) \\ Dr. Rosa Garcia Rodríguez \\ (board member) \\ Dr. Mónica Antón Gironés \\ (board member) \\ Dr. Maria Eugenia Sanchís Merino \\ (board member)
}

\author{
Dr. Fernando Florido López \\ (board member) \\ Dr. Virginia Rodríguez Vázquez \\ (board member)
}

\footnotetext{
doi: 10.18176/jiaci.0267
} 


\section{Introduction}

The primary mission of the Sociedad Española de Alergología e Inmunología Clínica (Spanish Society of Allergy and Clinical Immunology; Spanish abbreviation, SEAIC) was defined in the 2011-2018 SEAIC Strategic Plan (Olaguibel JM 2011) as "the diffusion and generation of knowledge centered on excellence in clinical practice, setting nationwide standards so as to become an indispensable interlocutor for public and private stakeholders" (translated from the original Spanish text). Clinical practice of this nature must be centered on the needs of patients and the population at large, and must ensure the safety of these individuals and the quality of care delivered for them.

The aim of this document is to provide a series of guidelines enabling clinical practice in allergy medicine to be carried out in accordance with these standards of quality and safety. Toward this end, these guidelines establish the structural requirements, human resources, and allotted times necessary to carry out these activities.

Allergic diseases encompass manifold clinical situations, spanning conditions of mere discomfort through severe reactions that may place the life of a patient at risk, sometimes with fatal outcomes. As the most severe allergic reactions are frequently provoked by food or drug allergens, the diagnostic procedures involving these allergens should be given high-risk status. Additionally, certain therapy procedures such as allergen immunotherapy or the administration of biological agents also have potential to induce a severe adverse effect.

In addition to clinical severity, other factors that should be taken into account when delivering patient care are both the impact that the disease has on the quality of patient's lives in both personal and social terms, as well as the direct and indirect costs deriving from these situations. Such costs are particularly significant in developed countries, where the incidence of allergic diseases is higher; indeed, this cost amounts to between 55 and 151 billion euros per year according to figures published by the European Academy of Allergy and Clinical Immunology (EAACI).

The gradual rise in allergic diseases seen in our society has been linked to changes in lifestyle associated with socioeconomic development, thus making primary prevention a complex undertaking. Nonetheless, secondary prevention aimed at early detection of the disease and tertiary prevention that seeks to forestall the development and progression of allergic manifestations are often viable, and require identification of the culprit agents, making the identification of these agents the key to allergy diagnosis.

In the case of allergy or hypersensitivity to drugs and foods, patients who consider themselves to be allergic to such substances usually avoid them, thereby creating a need to use alternative products; in the case of drugs, these substitute products may be more costly, less effective, or entail a greater risk of adverse effects.

It therefore seems important to reach a definitive allergy diagnosis in order to establish the appropriate preventive measures. Classification errors committed when judgment is based solely on anamnesis may have an impact on a patient's treatment options, potentially causing greater harm than would have occurred had an exhaustive allergy study been carried out to test for allergic response to drugs or food or to screen for respiratory allergy. Depending on the particular characteristics of a case, the clinical tools used to reach a definitive diagnosis are a comprehensive medical history, standardized skin tests, reliable in vitro testing, and controlled challenge tests to confirm a diagnosis or verify tolerance.

In general, there are two levels of intervention:

- Diagnosis (i.e. Identification of allergic patients and the search for management options)

- Therapy (i.e. procedures of desensitization, immunotherapy, or biological treatment)

While the risks inherent to the diagnostic procedures and treatment measures applied in routine clinical practice depend on a variety of factors that will be detailed in their corresponding sections below, the most noteworthy can be summarized as follows:

- The type of hypersensitivity reaction;

- Patient characteristics and comorbidities;

- The health professionals taking part in the care processes;

- The features of the care facility where the patient receives allergy care; and

- The allergy procedure itself, that is, skin tests, controlled challenge tests or desensitization.

Thus, the staff, time, and technical resources required to practice allergy medicine with adequate safety and quality vary from one episode of care to another. This document intends to establish the requirements of each procedure according to the risks inherent to each, providing insight on how care activity should be adjusted to the particular characteristics of each patient and professional setting. This set of guidelines offers a description of the structural and human resources necessary to provide care for the most complex patients, perform the most technically demanding procedures, and carry out care tasks that require the most prolonged periods of observation.

The consensus definition of an allergy day hospital (herein, $\mathrm{ADH})$ used in this document is a facility that guarantees patient safety during procedures and in which patients enjoy a certain degree of comfort and convenience whenever the expected period of observation exceeds 2 hours or when the risk inherent to the procedure or derived from the baseline situation of the patient calls for additional precautionary measures.

\section{Methodology}

The following METHODOLOGY was used to gather data and draft the present document:

- Allergic diseases were identified and classified in accordance with the International Classification of Diseases (ICD-10) system (the previous version, ICD-9, was used for those conditions not included in ICD-10).

- The diagnostic procedures performed in allergology were identified and classified.

- Each procedure was classified by level of risk following a consensus decision reached by the committees of the SEAIC.

- Additionally, we reviewed the official Ministry of Health and Social Services document setting forth the regulations for day hospitals. 
- The criteria appearing in the aforementioned document were adjusted for the specialty of allergology.

- The structural and human resources necessary for the practice of allergy were defined for all procedures.

- The times required for care staff and patients to properly perform the aforementioned procedures were established.

- Either because of a lack of a clear definition regarding these matters or the absence of existing indications in the scientific literature, the Delphi method was used to arrive at a consensus on those aspects in which differences in opinion arose (see Appendix III).

- The final document was drafted and revised by all authors

\section{Procedures in allergy}

\subsection{Conditions treated within the specialty of allergology (EUMS, NCMS) and coded under CIE-10}

J30 Vasomotor and allergic rhinitis

- J30.0 Vasomotor rhinitis

- J30.1 Allergic rhinitis due to pollen

- J30.2 Other seasonal allergic rhinitis

- J30.3 Other allergic rhinitis

- J30.4 Allergic rhinitis, unspecified

J31 Chronic rhinitis, nasopharyngitis, and pharyngitis

J32 Chronic sinusitis

J33 Nasal polyp

H10 Conjunctivitis

J45 Asthma

- J45.0 Predominantly allergic asthma

- J45.1 Nonallergic asthma

- J45.8 Mixed asthma

- J45.9 Asthma, unspecified (Late-onset asthma. Asthmatic bronchitis NOS)

J46 Status asthmaticus (Acute severe asthma)

J67 Hypersensitivity pneumonitis due to organic dust (Includes: allergic alveolitis and pneumonitis due to inhaled organic dust and particles of fungal, actinomycetic, or other origin).

- J67.0 Farmer lung (Moldy hay disease. Harvester and haymaker lung)

- J67.1 Bagassosis

- J67.2 Bird fancier lung

- J67.3 Suberosis

- J67.4 Maltworker lung

- J67.5 Mushroom-worker lung

- J67.6 Maple-bark-stripper lung

- J67.7 Air-conditioner and humidifier lung

- J67.8 Hypersensitivity pneumonitis due to other organic dusts

- J67.9 Hypersensitivity pneumonitis due to unspecified organic dust

J68 Respiratory conditions due to inhalation of chemicals, gases, fumes, and vapors

J69 Pneumonitis due to solids and liquids

J70 Respiratory conditions due to other external agents

D72.1 Eosinophilia

J82 Pulmonary eosinophilia, not elsewhere classified
B44 Eosinophilia due to aspergillosis J70.2-J70.4 Eosinophilia due to drugs

B50-B83 Eosinophilia due to specified parasitic infection M30.1 Polyarteritis with lung involvement [Churg-Strauss] T88.6 Anaphylactic shock, due to adverse effect of correct medicinal substance properly administered

T88.7 Unspecified adverse effect, due to a drug or medicinal substance

T78.0 Anaphylactic shock, due to adverse reaction to food T78.1 Food allergy, not elsewhere classified. Excludes: - dermatitis due to food (L27.2)

- in contact with the skin (L23.6, L24.6, L25.4)

T78.2 Anaphylactic shock, unspecified

T78.3 Angioneurotic edema

T78.4 Allergy, unspecified

D80-D89 Immunodeficiency

- D80 Immunodeficiency with predominantly antibody defects

- D81 Combined immunodeficiencies

- D82 Immunodeficiency associated with other major defects

- D83 Common variable immunodeficiency

- D84 Other immunodeficiencies

- D84.1 Defects in the complement system C1 esterase inhibitor [C1-INH] deficiency

L50.9 Urticaria

- L50.0 Allergic urticaria

- L50.5 Cholinergic urticaria

- T78.3 Urticaria with angioneurotic edema

- L50.8 Chronic urticaria

- L50.3 Dermatographic urticaria

- T78.3 Giant urticaria

- L50.1 Idiopathic or nonallergic urticaria

- L50.8 Recurrent periodic urticaria

- Q82.2 Urticaria pigmentosa

- L50.6 Contact urticaria

- L56.3 Solar urticaria

- T80.6 Serum reactions

- L50.2 Urticaria due to cold or heat

- L50.4 Vibratory urticaria

L20 Atopic dermatitis

L23 Allergic contact dermatitis

ICD-9-CM classification for drug allergy:

V14 Personal history of allergy to medicinal agents

- V14.0 Penicillin

- V14.1 Other antibiotic agent

- V14.2 Sulfonamides

- V14.3 Other anti-infective agent

- V14.4 Anesthetic agent

- V14.5 Narcotic agent

- V14.6 Analgesic agent

- V14.7 Serum or vaccine

- V14.8 Other specified medicinal agents

- V14.9 Unspecified medicinal agent

ICD-9-MC classification for food and insect allergies:

V15 Other personal history presenting hazards to health

- V15.0 Allergy, except allergy to medicinal agents

- V15.01 Allergy to peanuts

- V15.02 Allergy to dairy products 
- Excludes: lactose intolerance

- V15.03 Allergy to eggs

- V15.04 Allergy to seafood

- V15.05 Allergy to other foods

- V15.06 Allergy to insects and arachnids

- V15.07 Allergy to latex

- V15.08 Allergy to radiographic dye. Contrast media used for diagnostic $\mathrm{x}$-ray procedures

- V15.09 Other allergy, other than to medicinal agents

* Not all of the pathologies appearing in this list are specifically allergic phenomena. Some are the cause of symptoms typically found in allergic diseases.

\subsection{Portfolio of allergology procedures and activities}

The care services that form part of the specialty are grouped according to the following areas:

CODE 1. Inpatient care

1.1 Availability of inpatient care

1.1.1 Number of beds shared with another specialty or otherwise available, but not directly assigned to allergy

1.1.2 Number of beds assigned solely to allergy

1.2 Inpatient consultations

1.3 Day hospital

1.3.1 Day hospital (exclusively allergy)

1.3.2 Day hospital (shared)

CODE 2. Outpatient visits

2.1 Initial visit

2.2 Follow-up visit

2.3 All-in-one, high-resolution outpatient visit

2.4 Over-the-phone care

2.5 E-appointment

2.6 Patient-education appointment

CODE 3. Teaching

3.1 Graduate-level training

3.1.1 Residency training, allergy

3.1.2 Residency training, other specialties

3.2 Undergraduate training

3.2.1 Undergraduate medical students (years 3, 4, 5, 6)

3.2.2 Undergraduate nursing students

3.2.3 Students in nurse-assistant program

3.2.4 Laboratory technician

3.3 Fellows

3.4 Accredited continuous medical education

3.5.1 Continuous professional development (CPD) program

3.5.2 Primary-care courses

3.5.3 Courses for allergists and accredited sessions

3.5 Continuous training $\mathrm{W} / \mathrm{O}$ accreditation

3.5.1 Training activities for patients

CODE 4. Skin tests

4.1 Prick or intraepidermal test

4.1.1 Commercial extracts (aeroallergens, foods, venoms, occupational agents, etc.)

4.1.2 Foods or other homemade extracts (prick-prick)

4.1.3 Medicinal products

4.1.4 Others (occupational allergens, etc.)
4.2 Intradermal tests

4.2.1 Medicinal products

4.2.2 Hymenoptera (venom)

4.2.3 Autologous serum skin test

4.2.4 Cell-mediated immunity test

4.2.5 Fungi

4.3 Patch tests

4.3.1 Standard battery and specific commercial kits

4.3.3 Patch tests with homemade extracts (occupational, others)

4.3.4 Patch tests with medicinal products

4.3.5 Patch tests with foods

4.3.6 Photopatch test

4.3.7 Others

4.4 Skin tests for physical urticaria

4.4.1 Ice cube test

4.4.2 Dermographometer

4.4.3 Heat test

4.4.4 Pressure test

4.4.5 Temptest $\AA$

4.4.6 Others

CODE 5. Controlled provocation tests

5.1 Drug-provocation tests

5.1.2 Drug-provocation tests-ORAL

5.1.3 Drug-provocation tests-PARENTERAL

5.1.4 Drug provocation tests-OTHER

5.2 Food provocation tests

5.2.1 Tests for intolerance to foods

5.2.2 Controlled provocation with foods

5.2.3 Other challenges/provocations with foods

5.3 Conjunctival provocation test

5.4 Skin provocation/handling test

5.5 Insect-sting challenge

CODE 6. Nasal assessment

6.1 Nasal examination

6.1.1 Anterior rhinoscopy

6.1.2 Nasal endoscopy

6.1.3 Other diagnostic tests

6.2 Nasal obstruction test

6.2.1 Peak inspiratory flow rate (PIFR)

6.2.2 Rhinomanometry

6.2.3 Passive rhinomanometry

6.2.4 Active rhinomanometry (anterior-posterior)

6.2.5 Acoustic rhinometry

6.3 Assessment of symptoms

6.3.1 Predefined scoring based on symptoms

6.3.2 Visual analog scale (VAS)

6.3.4 Other scales

6.4 Nasal provocation test

6.4.1 Aeroallergens

6.4.2 Medicinal products (lysine-acetylsalicylate)

6.4.3 Occupational allergens

6.5 Assessment of nasal inflammation

6.5.1 Nasal cytology

6.5.2 Nasal nitric oxide

6.5.3 Inflammatory mediators: ECP and others

6.6 Olfactometry

CODE 7 Pulmonary examination

7.1 Methods of lung-function assessment 
7.1.1 Baseline spirometry

7.1.2 Flow-volume curve

7.1.3 Bronchodilator test

7.1.4 Lung-volume measurement and lung diffusion

7.1.5 PEFR

7.1.6 Impulse oscillometry

7.2 Assessment of bronchial inflammation

7.2.1 Bronchial FeNO

7.2.2 Exhaled breath condensate

7.2.3 Induced sputum

7.2.4 Sputum cytology

7.2.5 Measurements of inflammatory mediators in the airways

7.3 Bronchial challenge tests

7.3.1 Nonspecific bronchial challenge

7.3.1.1 Direct challenge with bronchoconstrictor agents

7.3.1.1.a Methacholine challenge

7.3.1.1.b Histamine challenge

7.3.1.2 Indirect challenge with bronchoconstrictor agents

7.3.1.2.a Adenosine monophosphate (AMP) challenge

7.3.1.2.b Mannitol challenge

7.3.1.2.c Exercise challenge

7.3.1.2.d Cold-dry air hyperventilation challenge

7.3.1.2.e Hypertonic (aerosol) saline challenge

7.3.2 Specific bronchial challenge

7.3.2.1 Environmental pulmonary allergens

7.3.2.2 Occupational allergens

7.3.2.3 Others (medicinal products, etc.)

\section{CODE 8 Therapy}

8.1 Allergen specific immunotherapy

8.2 Drug or food desensitizations

8.3 Biological treatments

8.4 Substitute treatments

8.5 Treatments of symptoms

8.6 Recommendations for allergic patients

\subsection{Allergy procedures classified by the resources required for delivery}

The scientific committees of the SEAIC, as bodies providing technical and care-delivery support for the practice of allergology in Spain, have proposed a three-level system for classifying the procedures performed within the field based on the estimated theoretical risk level of each. Each level contains a definition of the technical, structural, and human resources necessary for these procedures to be performed at optimal levels of safety and quality. Further indication is given regarding the time required for medical personnel to perform these tasks and how this time period relates to the total procedure duration, including times allotted for waiting and observation (Table 1). The levels are as follows:

Level A. Set of tests that meet the following criteria:

- Low complexity

- Short duration (the patient must remain under observation for a period of less than 2 hours,
Table 1. Levels of care for allergology procedures

\begin{tabular}{|c|c|}
\hline Level & Minimum requirements \\
\hline A & Basic care level \\
\hline $\mathrm{B}$ & $\begin{array}{l}\text { ADH (within the hospital or outside) with capacity } \\
\text { to perform emergency patient care and transfer in } \\
\text { less than } 15 \text { minutes. }\end{array}$ \\
\hline $\mathrm{C}$ & $\begin{array}{l}\text { ADH located within a hospital facility with } \\
\text { immediate access to the ICU, postoperative recovery } \\
\text { unit, or the emergency department or the } \\
\text { possibility of performing the procedure inside } \\
\text { the hospital facility. }\end{array}$ \\
\hline
\end{tabular}

independently of the time required for care delivery by medical staff)

- Low risk of allergic reaction

Tests at this level can be performed in any care facility providing basic medical services.

Level B. Set of tests that meet at least one of the following criteria:

- Greater complexity

- Estimated duration longer than 2 hours (time in which the patient must remain under observation by care staff, and independently of the time required for the procedure to be performed by medical personnel)*

- Moderate to high risk of reaction

(*) The definition of an ADH takes account of the time the patient must remain under observation, regardless of the risk involved in the test.

It is recommended that this type of test be conducted in an ADH, either inside or outside a hospital facility, provided emergency care is available and may be administered within a few minutes. Additionally, ICU care must be accessible no longer than $\mathbf{1 5}$ minutes.

Level C. Set of tests that meet at least one of the following criteria:

- High complexity

- Constant supervision and monitoring by nursing staff and/or specialist physicians

- Extreme risk owing to the potential seriousness of the allergic reaction or due to the presence of comorbidities.

These tests require hospital facilities equipped with monitoring systems and with direct access to the ICU/ postoperative recovery unit/emergency department, where a critical patient can receive urgent care if necessary, including immediate advanced CPR.

Table 2 lists the primary diagnostic and treatment procedures in allergy according to their theoretical levels of risk.

\subsubsection{Concepts regarding types of facilities}

- Testing Room. A space equipped with the goods and consumable material necessary to conduct basic studies within the specialty of allergy and which do not entail any risk: procedures classified as Level A and/or those having a short duration (under 2 hours).

- Allergy Day Hospital (ADH). A facility in which it is recommended to deliver allergy diagnosis or therapy when 
Table 2. Care services by theoretical level of risk

\begin{tabular}{|c|c|c|}
\hline Level A & Level B & Level C \\
\hline $\begin{array}{l}\text { Skin tests: intraepidermal and intradermal } \\
\text { (except in patients with previous } \\
\text { anaphylaxis or comorbidities*) }\end{array}$ & $\begin{array}{l}\text { Intraepidermal and intradermal tests in } \\
\text { patients with a history of anaphylaxis } \\
\text { or comorbidities* }\end{array}$ & $\mathrm{n} / \mathrm{a}$ \\
\hline Patch tests & $\mathrm{n} / \mathrm{a}$ & $\mathrm{n} / \mathrm{a}$ \\
\hline $\begin{array}{l}\text { Baseline spirometry and } \\
\text { bronchodilation test. }\end{array}$ & $\begin{array}{l}\text { Nonspecific bronchial challenge } \\
\text { (methacholine, mannitol, adenosine). }\end{array}$ & $\mathrm{n} / \mathrm{a}$ \\
\hline Impulse oscillometry. & Exercise challenge. & \\
\hline Peak flow measurement. & Specific bronchial challenge (HMW and LMW). & \\
\hline $\begin{array}{l}\text { Measurement of fractional concentration } \\
\text { of exhaled nitric oxide (FeNO) }\end{array}$ & Induced sputum study. & $\mathrm{n} / \mathrm{a}$ \\
\hline $\begin{array}{l}\text { Nasal endoscopy. } \\
\text { Nasal cytology. }\end{array}$ & Nasal biopsy. & $\mathrm{n} / \mathrm{a}$ \\
\hline $\begin{array}{l}\text { Nasal function examination: } \\
\text { Rhinomanometry and/or acoustic. } \\
\text { rhinometry }\end{array}$ & $\begin{array}{l}\text { Specific nasal challenge (with aeroallergens } \\
\text { or drugs, including LAS) and } \\
\text { nonspecific challenge. }\end{array}$ & $\mathrm{n} / \mathrm{a}$ \\
\hline Olfactometry & $\mathrm{n} / \mathrm{a}$ & $\mathrm{n} / \mathrm{a}$ \\
\hline PIFR measurement & $\mathrm{n} / \mathrm{a}$ & $\mathrm{n} / \mathrm{a}$ \\
\hline Nasal nitric oxide & $\mathrm{n} / \mathrm{a}$ & $\mathrm{n} / \mathrm{a}$ \\
\hline Specific conjunctival provocation. & Specific conjunctival provocation in patients at risk. & $\mathrm{n} / \mathrm{a}$ \\
\hline $\begin{array}{l}\text { Tests of physical urticaria: } \\
\text { Dermographism } \\
\text { Pressure } \\
\text { Temperature } \\
\text { Cold }\end{array}$ & $\mathrm{n} / \mathrm{a}$ & $\mathrm{n} / \mathrm{a}$ \\
\hline Autologous serum skin test. & $\mathrm{n} / \mathrm{a}$ & $\mathrm{n} / \mathrm{a}$ \\
\hline Latex handling test & Latex handling test in patients at risk & $\mathrm{n} / \mathrm{a}$ \\
\hline $\mathrm{n} / \mathrm{a}$ & $\begin{array}{l}\text { Controlled challenge test in the following circumstances: } \\
\text { - Non-immediate reactions }(>24 \mathrm{~h}) \text { (except in the } \\
\text { presence of comorbidities*). } \\
\text { - Immediate reactions }(<1 \mathrm{~h}) \text {, or latency periods of } 1-24 \mathrm{~h} \text {, } \\
\text { except in cases of extreme risk or comorbidities } \\
\text { - Non-immediate reactions }(>24 \mathrm{~h}) \text { in patients with } \\
\text { comorbidities* } \\
\text { - Controlled challenge test with contrast agents. }\end{array}$ & $\begin{array}{l}\text { Controlled challenge } \\
\text { test in patients at } \\
\text { extreme risk or } \\
\text { with comorbidities*. }\end{array}$ \\
\hline $\mathrm{n} / \mathrm{a}$ & Desensitization to medicinal products. & $\begin{array}{l}\text { Desensitization to } \\
\text { medicinal products in } \\
\text { patients at extreme risk } \\
\text { or with comorbidities*. } \\
\end{array}$ \\
\hline $\mathrm{n} / \mathrm{a}$ & Provocation with foods and additives. & $\begin{array}{l}\text { Provocation with foods } \\
\text { in patients at extreme } \\
\text { risk or with comorbidities }\end{array}$ \\
\hline $\mathrm{n} / \mathrm{a}$ & OTI with foods & OTI in high-risk patients. \\
\hline $\mathrm{n} / \mathrm{a}$ & Administration of biological drugs. & $\mathrm{n} / \mathrm{a}$ \\
\hline \multirow[t]{2}{*}{$\begin{array}{l}\text { Subcutaneous and sublingual } \\
\text { conventional immunotherapy. }\end{array}$} & $\begin{array}{l}\text { Cluster immunotherapy (aeroallergens and } \\
\text { Hymenoptera) (case-by-case basis). }\end{array}$ & $\begin{array}{l}\text { Hymenoptera-venom } \\
\text { immunotherapy in } \\
\text { patients at risk due to } \\
\text { comorbidities* }\end{array}$ \\
\hline & & $\begin{array}{l}\text { Experimental } \\
\text { immunotherapy with risk. }\end{array}$ \\
\hline $\mathrm{n} / \mathrm{a}$ & Hymenoptera-sting challenge. & $\begin{array}{l}\text { Sting challenge in patients at } \\
\text { risk due to comorbidities* }\end{array}$ \\
\hline Self-administration of treatment. & $\mathrm{n} / \mathrm{a}$ & $\mathrm{n} / \mathrm{a}$ \\
\hline Patient education. & $\mathrm{n} / \mathrm{a}$ & $\mathrm{n} / \mathrm{a}$ \\
\hline
\end{tabular}

Anaphylaxis: Previous anaphylaxis induced by the drug under study or analogs; ${ }^{*}$ Comorbidities: Comorbidities increasing the risk of a reaction (cardiac disease, use of beta-blockers and angiotensin-converting enzyme inhibitors) or patients with mastocytosis; Patients at extremely high risk due to extremely severe reactions or comorbidities that increase the risk of severe anaphylaxis; DPT: drug provocation test; OTI: oral tolerance induction; n/a: Not applicable. 
these activities have an estimated duration of 2 hours or more or have a degree of risk that makes it impossible to perform them in a testing room or ordinary examination room, though which do not justify hospital admission.

- Respiratory Function Testing Room. A space equipped with the goods and consumable material necessary to conduct those respiratory function tests that do not create a risk of fatality for the patient or do not take under 2 hours. For functional tests lasting for longer periods or involving greater degrees of risk, functional testing rooms should be part of an $\mathrm{ADH}$, as in an $\mathrm{ADH}$ latency periods are recommended to be observed between successive tests.

\subsection{Consensus reached using the Delphi method regarding the risk involved when performing the most common allergy procedures (see the criteria used for this purpose in Appendix III)}

- A low-risk food challenge is that which has a low risk of causing an allergic reaction and one in which the risk of a severe reaction is low ( $97.39 \%$, consensus).

- A moderate-risk food challenge is that which has a low or a high risk of causing a reaction and one in which the risk of a severe reaction is low ( $83.01 \%$, consensus).

- A high-risk food challenge refers to any test that involves a high risk of a severe allergic reaction $(94.97 \%$, consensus).

- A patient at high risk of developing a reaction to a food is considered to be one having a past history of allergic reaction to the culprit food over the past 6-12 months or who has had unfavorable results in allergy tests (i.e. skin test or highly positive IgE or sensitization to allergen components that are strongly associated with clinical relevance, such as ara h 2) (93.46\%, consensus).

- A patient at low risk of developing a severe reaction to a food is defined as one who has never had a severe reaction and does not have asthma. Additionally, the food involved in the study is not usually related to anaphylactic reactions or don't have a molecular sensitization profile linked to severe reactions $(97.39 \%$, consensus).

- Patients at high risk of a severe allergic reaction to a food are those with one or more of the following: a past history of a severe reaction, asthma diagnosis, a previous moderate/severe reaction to small amounts of the food, or certain conditions that may hamper treatment in the event of anaphylaxis (e.g. cardiovascular disease, difficult vascular access or intubation, beta-blocker medication use); additionally, a patient is considered to be at high risk if the food being studied is frequently implicated in severe reactions (e.g. peanut, nuts, fish, seafood, seeds) or if the patient is sensitized to allergen components associated with severe reactions ( $94.97 \%$, consensus).

\subsection{Consensus recommendations reached using the Delphi method regarding the facilities where the most commonly performed allergy procedures should be carried out}

- Regardless of the expected severity of the reaction, it is recommended that all provocation challenges with food be performed in an $\mathrm{ADH}$ due to the duration of the test (73.86\%, majority without consensus).

- Challenges with foods that pose a moderate risk require an $\mathrm{ADH}$ (90.20\%, consensus).

- To carry out a moderate-risk food challenge, a dayhospital unit is essential, and must be located within a hospital facility $(83.01 \%$, majority without consensus).

- High-risk food challenges require an $\mathrm{ADH}$, which should be located within a hospital facility ( $95.53 \%$, consensus).

- Desensitization to food allergens and controlled challenge tests have an extremely high level of risk and high complexity, due either to the severity of the allergic reaction or because of existing comorbidities that increase the risk of anaphylaxis. These activities must be performed in an $\mathrm{ADH}$, though potentially supported by intensive care units ( $96.73 \%$, consensus).

- Controlled drug provocation tests in patients with suspected immediate reactions should be performed in an $\mathrm{ADH}$ regardless of the risk level of the procedure (94.12\%, consensus).

- Controlled challenge tests for non-immediate drug reactions should be performed in an $\mathrm{ADH}$ facility (73.20\%, majority without consensus).

- An ADH facility is recommended for drug-desensitization procedures (94.97\%, consensus).

- Desensitization to drugs and controlled provocation tests have an extremely high level of risk and high complexity, due either to the severity of the reaction or because of existing comorbidities that increase the risk of anaphylaxis. These procedures must be carried out in an ADH located within a facility that has an ICU/ postoperative recovery unit/emergency department, where patients who develop a severe reaction may be treated (98.04\%, consensus).

- Aminimum floor space of $2 \mathrm{~m}^{2}$ per patient is recommended for all provocation tests/desensitization/immunotherapy with foods for proper test administration and patient follow-up. (The calculation of floor space per patient refers to the total size of the room, so a $20 \mathrm{~m}^{2}$ space can be used for up to 10 patients $)(88.24 \%$, majority without consensus).

- An independent space for carrying out intervention-related treatment procedures (food-allergen desensitizationimmunotherapy) and monitoring-observation is recommended, though this area may be located within an $\mathrm{ADH}$ and consist of a bed or examination table sectioned off for the sake of privacy ( $92.81 \%$, consensus).

- A room for treating allergic reactions is recommended. It should be adjacent to the room where procedures are performed but out of sight of other patients in the ADH (94.77\%, consensus). Each of these stations should have a floor space of 4-5 $\mathrm{m}^{2}(92.81 \%$, consensus) and be specifically designed for this purpose $(98.69 \%$, consensus). A majority of surveyed professionals believe that the room for the treatment of positive reactions in the ADH should have 1 bed for every 10 patients devoted to diagnostic and treatment procedures $(85.62 \%$, majority without consensus).

- It inadvisable to perform intraepidermal or intradermal tests on patients with a history of anaphylaxis of grade 2 
or higher in specialist clinics located outside of a hospital facility when these lack access to an ICU/postoperative recovery unit/emergency department in under 15-30 minutes (83.66\%, majority without consensus).

\subsection{Guidelines on the duration of medical and nursing appointments by clinical procedure (partial application of the Delphi methodology for consensus)}

- It is recommended that an initial allergy visit have an average length of 30 minutes (96.08\%, consensus).

- For all-in-one, high-resolution outpatient clinic visits it is recommended to allot 45 minutes for appointments with the allergist ( $94.12 \%$, consensus).

- It is recommended that follow-up allergy appointments have an average length of 15 minutes $(90.20 \%$, consensus).

- Remote consultation (by telephone or e-appointment): 15 minutes

- Patient education: 15-30 minutes

- Time devoted to training: 70 hours per year

- Time devoted to research activities: 4-8 hours per week

\subsection{Consensus, via the Delphi method, on the administration of allergen immunotherapy}

Parallel to the aforementioned guidelines, and also using the Delphi method, the SEAIC Immunotherapy Committee has reached a consensus on the conditions for administration of allergen immunotherapy.

\section{Minimum standards for each risk level}

\subsection{Structural resources and equipment for Level $A$}

\subsubsection{Physical space}

Administrative area or reception desk

Area devoted to patient intake, coordinating the movement of patients throughout the area containing the examination rooms and spaces devoted to other procedures. Additionally, this unit manages appointments, carries out general administrative tasks, and organizes care delivery. The requirements for this area include a computer with Internet access where there is an electronic health record (EHR) system, printer, and a telephone.

Waiting room

It is recommended that the space devoted to the waiting room measure at least $8 \mathrm{~m}^{2}$, though this waiting room may be shared with other departments requiring a space for this purpose.

Examination room

Physicians' offices should have independent spaces to guarantee privacy during doctor-patient interviews. These rooms should be furnished with a desk for conversations with patients, two visitor chairs, an examination table, and a sink or alcohol-based hand sanitizer. These rooms may be used for other activities as well.

If the facility uses an EHR system, the examination room should be equipped with a computer with Internet access, a printer, and a telephone.
Nursing or diagnostic procedures area (testing room)

An area used to perform diagnostic procedures and treatment of allergic reactions, in adherence of the specifications appearing below.

While the size of the room will depend on the number of patients receiving treatment at the same time within this space, the minimum recommended size is $15 \mathrm{~m}^{2}$. If the room is to be used to examine both children and adults, it is recommendable to separate these two spaces; additionally, for interventions in patients of pediatric age, the space must be designed to accommodate an individual accompanying the patient. Where EHRs are used, there must be a means of transcribing the information gathered during studies of patients. For paperbased EHRs, the necessary material must be available, such as cellophane wrap or transparent sheets, data collection forms, etc. for transcription.

It is recommended that testing rooms be equipped with the following:

- Reclining chairs or, where these are not available, comfortable chairs

- Chairs for individuals accompanying patients (children)

- Refrigerator

- Spirometer

- Desk

- Closet or glass cabinet for storing material

- Computer, printer, and telephone

- Sink

Samples for in vitro studies may be extracted in the service; alternatively, patients may be transferred to the medical center's centralized extraction unit. Where extractions are performed in the allergy service, all consumable material required should be on hand, including needles, syringes, collection tubes, and materials necessary for a coding system with which to identify the samples.

Treatment area (reactions)

It is recommended that there be an area to treat adverse reactions situated in the proximity of the space where diagnostic procedures are performed, as even the procedures with the lowest level of risk may provoke an allergic reaction requiring medical attention. The set of indispensable, basic equipment for this room is as follows:

- An examination table - with wheels, if possible - to be used to transport patients; this table must be suitable for performing CPR

- Oxygen-supply equipment and vacuum source

- Crash cart with available defibrillator

- Blood-pressure monitor

- Pulse oximeter

- Consumable supplies to treat adverse reactions (e.g. drugs, syringes, needles)

- Availability of an ECG system (this may be located in the vicinity of the unit).

\section{Restrooms}

Restrooms may be exclusively for the unit or shared with other hospital services; if shared, however, they should be located on the same floor as the allergy department and have no architectural barriers impeding access by people with disabilities. 


\subsection{Structural resources and equipment for Levels $B$ and C: Allergy Day Hospital (ADH)}

Many of the tests conducted within the field of allergy have characteristics that qualify them to be carried out in an allergy day hospital (ADH). These test characteristics are as follows:

- Longer duration (over 2 hours), requiring that the patient remain in the facility under supervision for this extended period of time, though without requiring hospitalization.

- Theoretical risk of developing a potentially fatal adverse reaction; the prospect of such a reaction requires monitoring and rapid recognition of the reaction, thus creating the need for active observation and, where necessary, a readiness to respond swiftly.

The difference between Levels B and C is linked to the proximity of the ICU/postoperative recovery unit/emergency department and the possibility of performing procedures of the highest risk level within the ICU/postoperative recovery unit/ emergency department. An ADH that provides care for Level B diseases may be located outside of the hospital, though these facilities must enable ICU access in under 15 minutes. Level $\mathrm{C}$ procedures must be performed within a hospital facility.

\subsubsection{Definition of an ADH}

A "day hospital" refers to care delivered in a hospital over a number of hours. This care may consist of a multitude of diagnostic procedures, clinical studies, and/or examinations or treatments that cannot be performed in an ordinary outpatient clinic though do not justify conventional admission on an inpatient basis. The main types of activity that take place in an $\mathrm{ADH}$ are allergology-related procedures that entail a greater risk or take longer.

This document aims to provide public-health authorities, administrators in the public and private sector, and health professionals with a series of criteria for the continued introduction of ADHs, setting forth recommendations on the physical resources and staffing necessary for these units to function properly. The information offered herein may contribute to efforts to improve the safety and quality in allergy medicine in all its dimensions, including the efficiency of care delivery.

Special emphasis is given to certain imperatives where this heightened level of importance is dictated by regulatory requirements or where the body of evidence backing these recommendations is of sufficient solidity so as to cause the group of experts who authored this document to highlight them accordingly.

\subsubsection{ADH: Current Situation}

The day hospital is a means of organizing and managing a particular set of care services, known as the service portfolio or portfolio of services. To carry out these activities, a day hospital requires certain structural and functional conditions to ensure the efficiency and quality of the services while guaranteeing the safety of those who use them.

Assigned the code U65 under Royal Decree 1277/2003, day-hospital units have undergone substantial development in both the public and private health sectors in recent years, benefiting both the health facilities themselves as well as numerous patients who have received care in these facilities.
This document adopts the definition of a day hospital that appears in the aforementioned Royal Decree, which is as follows: a care unit where, under the supervision or at the indication of a specialist physician, treatment or care is rendered for patients who must undergo diagnostic or therapy measures that require continuous attention from a physician or nursing professional over a number of hours, but do not require hospital admission.

Given the lack of materials on the topic of ADHs, the present document has been drafted to provide support to allergy services and units, informing them of the recommendations for each procedure in light of the risk involved in each. All aspects regarding the physical resources, staffing needs, and estimated length of procedures that were met with differences in opinion were handled by applying the Delphi method in search of a consensus.

As seen in the most recent data published in the Alergológica study, $56 \%$ of participating facilities have an $\mathrm{ADH}$ in their center. The median number of patients receiving treatment in these centers was 424, though substantial variability was detected among these centers. With regard to the relation with critical-care units - this being the criterion used in this document to classify allergy-care procedures as appropriate for a Level B or C facility $-39 \%$ of facilities conduct procedures inside the health center's ICU, while these procedures take place in rooms located adjacent to the ICU in $42 \%$ of the participating centers.

\subsubsection{Factors involved when operating an $A D H$}

The following matters are of relevance:

a. Patient rights and guarantees;

b. Patient safety;

c. Organization and management of the ADH;

d. Physical structure and material resources required by the unit;

e. Staff; and

f. Care quality.

a) Patient rights and guarantees

Patient rights granted under health-care legislation must be respected and upheld in all medical specialties, and safeguarding these rights is mandatory for both the ADH as a unit and the allergist as a professional. This section contains a detailed account of the information to be made available to patients - as well as family members and carers - within this modality of care delivery as well as a general overview of other legal matters to be taken into account in day hospitals of this nature and, where applicable, the health facilities where these day hospitals are located. Overall, allergy is a discipline with a wealth of information on the procedures to be undertaken prior to obtaining informed consent from patients. In addition to the matters on which we elaborate below, this issue of informed consent will be a core topic of the present section.

i. Information on the general characteristics of ADHs.

ii. Detailed information on the disease process and procedures used in an $\mathrm{ADH}$ for diagnosis and treatment.

iii. Informed consent.

iv. Instructions and recommendations. 
i. General characteristics of an ADH. Most patients who visit an ADH do so on an elective basis, and a majority report to the facility in the company of another person. (For minors, caregiver accompaniment is compulsory.) The services rendered in an $\mathrm{ADH}$ must limit the extent to which they disturb the daily lives of patients and their family members and carers. The information provided by an ADH should include all aspects that may facilitate the support role played by carers, promoting active collaboration by these individuals in patient care.

Patient welcome guides and other materials provided to patients on intake (i.e. pamphlets, leaflets) should include, at minimum, a description of the different phases of care the patient will undergo as well as special recommendations and warnings. Photographs of the ADH facilities help patients familiarize themselves with the facility and increase their sense of comfort with their surroundings. Patients likely to receive treatment in an ADH can be given these materials during preliminary medical visits to the allergy department or be directed to a website containing this information.

An abridged version of this document may be distributed in the admissions/reception areas and waiting rooms of hospitals and primary-care centers to inform the general population of the existence of this care modality and the proximity of one such unit in their community.

ii. Detailed information on the disease process and procedures used in an ADH for diagnosis and treatment. Information provided for patients should be specific to a given treatment or diagnostic procedure and include information on the risks involved as well as their potential benefits.

iii. Informed consent. In accordance with applicable legislation, invasive diagnostic and treatment procedures as well as the administration of treatments that involve potential risks or discomfort and which may have a deleterious effect on the health of the patient require written patient consent before they are performed.

It is recommended that all diagnostic and therapy procedures of this nature included in the portfolio of services of the ADH have a specific informed-consent document.

iv. Instructions and recommendations. Patients should be given a welcome guide containing the following aspects in writing:

- Instructions on how to prepare for a visit to the ADH, such as whether they require or should avoid any type of treatment, whether they will need to provide information or bring any of their products to the facility, etc.

- Useful information for getting to the facility (e.g. time scheduled, contact person).

- Instructions to follow while in the ADH, including what they may and may not do while in the facility. For example, they may be encouraged to take reading material along with them, use headphones to listen to music, etc. Other aspects may include whether or not they need to or are advised to come accompanied, following rules in consideration of other users and employees of the service, and the like.

- What to do during the procedure itself (e.g. appearance of alarm symptoms).

- Instructions to follow on discharge, adapted for each procedure. This information should cover all incidents that may arise after the patient has returned to their home or alternative accommodations. Each potential incident must be paired with the medication and/or care that the patient must receive, as well as a telephone number they may call with questions.

Generally, ADHs are recommended to have the following documentation:

- Portfolio of services.

- Patient orientation plan.

- Code of ethics.

- Guides, pathways, or protocols used in clinical practice, with documented evidence.

- Written procedures in the case of clinical research.

- Health records.

- Previous instructions.

- Discharge reports.

- Protocols guaranteeing patient safety, confidentiality, and legally mandated access to patient information.

- Forms and procedures for grievances and suggestions.

\section{b) Patient safety}

Protecting the patient and guaranteeing their safety is the ultimate goal of an ADH. Procedure-specific issues will be detailed below, alongside the results obtained when the group of authors applied the Delphi method to measure consensus. Overall, a consensus opinion emerged, indicating that all procedures require the following:

- Standard operating procedures (SOP), including support for recording data relevant to the procedure while it is ongoing, such as vital signs, dose levels administered, etc. $(96.65 \%$, consensus).

- SOP for serious adverse reactions, available to all care staff $(96.09 \%$, consensus).

- Protocol for treating adverse reactions (with a code for cardiac arrest) (97.21\%, consensus).

- System for reporting incidents (94.97\%, consensus).

- Staff with specific training in CPR (98.32\%, consensus); for this purpose, it is recommended that these professionals undergo training every 2 to 3 years $(91.50 \%$, consensus).

Among other aspects that may play a role in maintaining quality and safety, one particularly relevant issue concerns identification measures:

i. Management of drugs

ii. Patient identification

i. Management of drugs. Especially when procedures are carried out to study potential allergic reactions to or tolerance of drugs or when specific drug treatments are administered to patients, ADHs shall have an established protocol for storing and monitoring the expiration/shelf life and packaging of pharmaceutical products. Additionally, this protocol shall guide the identification, handling, and prescription of these substances, placing particular emphasis on those that involve a high risk. Measures should be in place to monitor the temperature of drugs that have predetermined temperature requirements. When using split doses as part of a build-up controlled challenge, drugs must be identified through proper labeling of dosing so as to avoid any possible confusion.

ii. Patient identification. A reliable method must be in place to accurately identify patients before carrying out any 
procedures involving a degree of risk and prior to administering drugs or conducting any diagnostic procedure.

\section{c) Organization and management of the ADH}

The following requisites apply when organizing an $\mathrm{ADH}$ :

- A manual detailing the organizational planof the unit.

- The hierarchical structure of the ADH should be clearly defined, including all roles and reponsabilities.

- Comprehensive information for patients and their family members or carers should be available. An informedconsent document should be available, covering all aspects stipulated under legislation in force.

- Patients, their carers or other individuals accompanying patients should be treated with respect.

- Procedures should be planned and designed in order to minimize the risk of necessary inpatient admission.

- Direct responsibility over the ADH throughout the process, notwithstanding any support given to the unit from time to time from other levels of the health-care system (e.g. primary care, emergency department, conventional hospitalization units).

- Rigorous fulfillment of criteria for discharging patients.

- Detailed specification of care following discharge from the $\mathrm{ADH}$.

- Annual report of care results.

It is imperative that all ADHs have the following:

i. Admissions

ii. Documentation and health records

ii.a. Health records

ii.b. Record of patients treated

ii.c. Confidentiality of information

i. Admissions. The ADH admissions service may be part of the facility's general admissions desk or be exclusively devoted to the ADH. For public health centers, it is recommended that all aspects related to clinical documentation, patient registry, discharge reports, and data protection be directly linked to the hospital facility where the $\mathrm{ADH}$ is located.

ii. Documentation and medical records. Clinical documentation comprises all documents generated as part of the care process, whether in hard or soft copy and independently of the format in which they appear. This documentation includes the following: patient health records, informed consent, previous instructions (where applicable), as well as admission reports, referrals, and discharge reports.

Clinical documentation is to be managed by the admissions unit and the clinical documentation unit or equivalent division. Management of this documentation entails generation, custodianship, lending, duplication, copying, monitoring, and disposal of clinical documents of any type.

Clinical documentation must be kept in a condition that ensures proper maintenance and security for the time period appropriate for each situation; this time period shall not be shorter than 5 years as of the conclusion of each care process.

ii.a. Health records. Each patient must have their own personal health records, and these records are likely to be shared among staff members and health centers and across different levels of care. These records must comply with the technical specifications for compatibility set by each health service.
The care-related information appearing in patient health records may appear in hard copy or in soft copy (i.e. digital, electronic, or telematic media) provided they are retrievable and usable in their entirety. As for the design of these documents, their minimum required contents, as well as additional requirements, guarantees, and directives for their use, units shall uphold the provisions of Spanish Basic Law 41/2002 on the autonomy of the patient and the rights and obligations with regard to clinical information and documentation.

ii.b. Record of patients treated. Discharge report upon conclusion of the procedure is mandatory. Preliminary reports shall be issued, containing detailed information on the procedure. In case of a possible delayed reaction, the patient should receive information containing clear instructions on how to procede and monitor the reaction.

ii.c. Confidentiality of information. All patients have the right to confidentiality. The Spanish law regulates the autonomy of the patient and their rights and obligations.

d) Physical structure and material resources required by the unit

The following is required:

i. Functional needs assessment

ii. Economic study

iii. Specific areas

iv. Materials: medical equipment and supplies

i. Functional needs assessment. The functional program shall identify the needs-based criteria deriving from the procedures carried out under this modality of care delivery, and shall include the following:

- A demographic study of the surrounding area, with special focus on the patient population and the criteria used for patient selection.

- The projected demand for treatment in the ADH in light of the volume of procedures included in the portfolio of services. The assessment should take into account the unit's ability to draw patients from its surrounding area once it is fully operational.

- An analysis of the architectural characteristics of the space where the ADH will be housed, either by designing a new facility or renovating an existing one.

- Analysis of the output capacity of the unit, including minimum and maximum efficiency scenarios.

- A study of staffing and equipment needs as a function of demand, activity forecasts, and the existing portfolio of services in the hospital.

- Care flows and priority levels. Given that not all procedures performed within the specialty of allergy medicine will take place in this setting, it is important to establish the criteria to be applied for referring patients to the $\mathrm{ADH}$ rather than a room used for conventional testing. These criteria shall be based on the foreseeable risk of allergic reaction during the procedure.

ii. Economic study. The structure of an $\mathrm{ADH}$ is determined by its care-delivery function. It therefore should be taken into account that these units are largely self-sufficient, with patient intake, delivery of care, and discharge all occurring within the ADH facility. An ADH may also provide support for other hospital services requiring specific procedures for 
their patients, such as an emergency inter-hospital consultation regarding an inpatient whose treatment may be continued in the ADH.

The functional program of an ADH shall take into account the fact that procedures may be performed in different physical spaces, such as examination rooms, areas furnished with hospital beds or reclining chairs, or in rooms devoted to specific treatments. Patient recovery must take place in an area with space for beds or reclining chairs; these furnishings are most often distributed throughout a common-use room.

There is a wide range of possible functional configurations. Some facilities may use the same space for examination, treatment, and patient recovery or may opt to use different areas for these functions. By way of example, examination and diagnosis may be done in an examination room, treatment may be administered in a reclining chair, and patients recovering from their procedures may remain at a station located in an observation room.

\section{iii. Specific areas.}

The activities performed in an $\mathrm{ADH}$ require four spaces:

- A reception area, including a waiting room, can be shared with the rest of the allergy department, though within this area patients should be instructed to report to a specific area devoted to the $\mathrm{ADH}$.

- Care-delivery areas for visits and examinations. The physician offices belonging to the allergy department may be used when making first contact with patients, confirming the treatment to be administered, and checking clinical criteria, after which the process continues in the ADH.

- Nursing station and general support services. This is a specific area where nursing staff carry out tasks before and after interacting with patients (e.g. preparing doses).

All Group B allergy procedures must be conducted in a hospital setting or in a unit with rapid access to the hospital (under 15 minutes), as such facilities are outfitted with the necessary equipment and have the appropriate staff to intervene quickly, identifying and treating patients who develop a serious reaction.

The following additional considerations apply when procedures are performed in a specialist-care center:

- A support or referral hospital must be accessible should the need arise to transfer a patient for in-hospital care due to a reaction occurring after the patient has received treatment and where the patient may recover in the hospital's allergy department.

- An emergency telephone line (061/122 within Spain) and access to the allergy department, where a fully equipped ambulance may be summoned to evacuate patients in under 15 minutes.

iv. Materials: medical equipment and supplies. A comfortable and suitably sized bed or reclining chair.

A gurney to be used in case of patient evacuation due to an allergic reaction; these gurneys must be fully equipped and in adherence of official standards for performance of CPR.

Desk for the specialist physician. Desk for patient consultations, including one chair for the patient and another for the person accompanying them. It is recommended that this desk be outfitted with a computer with access to the electronic health record (EHR) system, provided such a system is used. In the absence of an EHR, access to printed health records at all times throughout the procedure. Cellular phone or land line.

Individual physician desks are not required, as another available desk in the department may be used for this purpose.

Work space for nursing staff. This space is used to store all information related to the procedure being performed, the outcome of the procedure, the treatments administered (if necessary), and the like. This area shall also contain documents on recommendations for before and after the procedure, information on appointment scheduling, etc.

\section{Chairs for patients}

Chairs for individuals accompanying patients. All minors must be accompanied by an adult. Optional for adult patients and depending on the size of the room used for diagnostic testing.

Refrigerator. This refrigerator need not be for exclusive use by the ADH, and may be used by another hospital service. A refrigerator must be used wherever medicinal products or other medical products requiring continuous refrigeration are administered. The health facility or the allergy department shall have a refrigerator with a thermometer indicating minimum and maximum temperatures, used specifically to conserve these medicines and other products. A designated individual shall be charged with overseeing this equipment.

Storage area. This area is to be used to store medication and other medical supplies.

Brand-name and generic medicinal products. All medications necessary to perform diagnostic techniques and treat any allergic reactions that patients may develop must be inventoried periodically to inspect their expiration dates.

Narcotic medication must be stored according to the control regulations of the health facility in which the ADH is located (for example, in a locked box or safe if these substances are stored in the allergy department or as indicated depending on the case).

Obligatory minimum medication:

- Epinephrine $1 \mathrm{mg} / \mathrm{mL} ; 1: 1000$.

- Naloxone hydrochloride $0.4 \mathrm{mg} / \mathrm{mL}$.

- Volume expanders (albumin, saline solution, Ringer's lactate, colloids).

- Sodium bicarbonate $4.2 \%$.

$-10 \%$ and $5 \%$ dextrose.

- Distilled water and saline for dilutions.

- Bronchodilators.

- Corticosteroids.

- Antihistamines.

- Glucagon.

All material required for the procedures performed.

Oxygen. The system for supplying oxygen may be centralized or an oxygen cylinder may be used, in which case the cylinder must be inspected periodically.

Crash cart. This cart should be used exclusively in the allergy facility and should be sealed and contain documentation listing all its components. It should be inspected periodically by designated staff members, who shall sign off following their inspection. It is recommended to have two carts - one for adults and another for children; otherwise, all material can be contained in the same cart, though material specially for use in children must be kept separate from the rest. 
Monitor or ECG. For procedures having the highest risk levels, it is recommended to have a monitor with which to record heart rhythm. This monitor should also record vital signs (i.e. ABP, HR, oxygen saturation). Where no monitor is available, it is recommended to have an ECG device in the unit or immediately accessible nearby as well as a blood-pressure monitor and pulse oximeter.

Defibrillator (available in the ADH proper or nearby)

Spirometer

Rhinoscope

Stethoscope

Flashlight

Tongue depressors

Forehead lamp

Scale and stadiometer

Indoor meteorological instrumentation (thermometermercury barometer-hygrometer to measure relative humidity)

Calibration syringe.

Rigid mouthpieces

Adapter for pediatric patients

Disposable filters

Transducers

Noseclip

Paper-based or digital means of recording procedures

Holding chamber

Other supplies: allergen extracts for skin tests, lancets, latex-free gloves, antiseptic solution (alcohol-based or other), blotting paper, razors for shaving, stopwatchtimer, receptacle for disposal of hazardous materials or biocontaminants, skin marker or pen, wheal-measurement device (other methods for collecting results from prick tests are optional).

Support services: central storeroom, purchasing, supply, and the admissions service.

Care services: ICU, emergency department, laboratory (the facility used by the health center to receive and process samples), diagnostic radiation service, pharmacy service (to prepare and dispense drugs for diagnosis and treatment).

It is essential for the facility to have easy access to a nearby hospital unit (ICU, postoperative recovery, emergency department) that is capable of treating patients in critical condition and administering advanced CPR. The physicians in this unit should be aware that the allergy department carries out high-risk procedures, and as a result their intervention may be required. Should the need arise, a direct line is to be available to request help; this shall be the same line used for this purpose throughout the facility. All ADH staff must know which number to call.

Cleaning, disinfection, and sterilization. The rules in place for the hospital facility where the ADH is located shall be followed.

\section{Care-activity recording systems}

Agenda. A specific daily list of appointments (paper or electronic) indicating the name and EHR number of the patients scheduled to be seen in the ADH for studies and treatment of allergic reactions.

\section{Nursing forms}

Forms for recording all nursing activity involved in the procedure (e.g. vital signs, doses administered).

\section{Protocols and procedures}

SOPs shall be in place for all activities performed when a patient undergoes a study or treatment for allergic reactions. These protocols shall be in writing (or in a computerized folder with password-protected access given to all members of the service). One of these protocols shall concern hand hygiene. This will be included as part of the basic set of documentation of the unit. It is recommended that each protocol have one or more leads (physician or, where a nursing protocol is in place, a nurse) and a date set for the document to undergo review.

Protocol for adverse reactions

A protocol shall be in place detailing the rapid-response actions to be taken in the event of an allergic reaction during a diagnostic or therapy procedure. The unit shall also have a protocol for treating such reactions (including a code for cardiac arrest).

\section{Incident-reporting systems}

The unit shall have a system for reporting incidents. In hospitals that use an EHR system, the unit may use the same procedures employed throughout the facility. Otherwise, the service should design its own system of reporting. In the absence of digital health-record system, paper-based documents shall be used. The service should hold periodic discussions on the reporting of incidents. A commission made up of representatives from all levels of the organization may be set up within the service to analyze all of the incidents that take place.

e) Staff

To function properly, an ADH must have the following:

i. Attending physician

ii. Nursing staff

iii. Assistant nurse

iv. Administrative staff

v. Other support staff

i. Attending physician. Each ADH room devoted to diagnosis and treatment should have its own assigned specialist available at all times. This specialist must be easily located and remain nearby, so as to guarantee proper supervision of the diagnostic tests and therapy interventions being carried out in the room as well as to ensure rapid response in case of reactions taking place during the procedures. Indeed, for all controlled challenge tests, it is recommended that there be an allergist either physically present or nearby $(98.8 \%$, consensus). Further, it is recommended that there be an allergologist physically present and whose sole responsibility is to oversee the $\mathrm{ADH}$, performing a maximum of 10 to 15 procedures per day ( $96.08 \%$, consensus).

ii. Nursing staff. Unlike other medical disciplines, in the field of allergy the most common diagnostic and therapy procedures are customarily performed by nursing staff with special training in these tasks.

Therefore, these nursing professionals must be present for the procedures administered in the $\mathrm{ADH}$, both while the procedure is taking place as well as to appropriately supervise the results of these procedures once they have been completed. Indeed, the presence of nursing staff is recommended for all controlled provocation tests ( $94.97 \%$, consensus). The number of professionals required will vary based on the 
volume of patients requiring attention. In optimal conditions, it is recommended that there be 2 nurses for every room, though this ratio may be reduced if there is a low volume of patients. Nursing staff shall be responsible for handling allergen extracts to ensure that these are properly conserved and inventoried, monitoring the expiration dates, overseeing supply management, and the like.

All units are recommended to hold training events and refresher courses so that staff may obtain and maintain accreditation in each of the procedures performed in the ADH.

It is desirable to allot a set amount of time in nurses' schedules for these professionals to conduct patient-education sessions, as training of this sort is highly necessary for individuals with allergies.

iii. Nursing assistant. Assistant nurses are increasingly more scarce outside of inpatient wards.It is recommended that each room devoted to diagnosing and treating allergic diseases be staffed by nurse assistants. These employees may take part in tasks concerning appointment scheduling and patient intake and identification. In the opinion of the allergists surveyed for these guidelines, it is advisable to have nursing assistants in allergy units to provide support ( $90.85 \%$, consensus).

iv. Administrative staff. ADHs located within the same facility as their affiliated general allergy departments may use

Table 3.

\begin{tabular}{|c|c|c|}
\hline \multicolumn{3}{|c|}{ Structure and function of the reception area } \\
\hline Area & Function & Structure \\
\hline ACCESS & $\begin{array}{l}\text { Facilitate the arrival of patients to the allergy } \\
\text { department and ADH }\end{array}$ & $\begin{array}{l}\text { Appropriate signage outside the facility } \\
\text { Access for people with disabilities }\end{array}$ \\
\hline ADMISSIONS & $\begin{array}{l}\text { Receiving patients: welcoming procedure } \\
\text { and intake }\end{array}$ & $\begin{array}{l}\text { Appropriately sized space } \\
\text { Guarantee a certain degree of privacy } \\
\text { Equipment: computer systems, telephone, fax, etc. }\end{array}$ \\
\hline & & $\begin{array}{l}\text { Easily identifiable from the moment patients arrive to } \\
\text { the floor where the unit is located }\end{array}$ \\
\hline WAITING AREA & $\begin{array}{l}\text { Provide patients and those accompanying them } \\
\text { with a space in which to wait until they are } \\
\text { called into the diagnosis or treatment area }\end{array}$ & $\begin{array}{l}\text { Comfortable seating } \\
\text { Restrooms located nearby } \\
\text { Cold water drinking fountain } \\
\text { Entertainment options: television, magazines, etc. }\end{array}$ \\
\hline RESTROOMS & $\begin{array}{l}\text { For use by patients and those accompanying } \\
\text { them while they wait }\end{array}$ & $\begin{array}{l}\text { Sink and toilet } \\
\text { The number of restrooms shall be proportional to the } \\
\text { capacity of the waiting room }\end{array}$ \\
\hline \multicolumn{3}{|c|}{ Structure and function of the ADH proper } \\
\hline Area & Function & Structure \\
\hline $\begin{array}{l}\text { EXAMINATION } \\
\text { ROOMS }\end{array}$ & $\begin{array}{l}\text { Examination, diagnosis, and clinical assessment } \\
\text { of patients; appropriateness of indication of the } \\
\text { procedure. Information and support. }\end{array}$ & $\begin{array}{l}\text { These spaces may be specific to the ADH or used for } \\
\text { other purposes as well. } \\
\text { Appropriate equipment: desk, rolling office chair, } \\
\text { chair for the patient and the individual accompanying } \\
\text { them, computer, and all other equipment needed for a } \\
\text { medical examination room. This room may be used by } \\
\text { nursing staff. }\end{array}$ \\
\hline $\begin{array}{l}\text { ADH CARE } \\
\text { STATION }\end{array}$ & $\begin{array}{l}\text { Used to conduct procedures and administer } \\
\text { treatment. }\end{array}$ & $\begin{array}{l}\text { Depending on the type of procedure and/or treatment, } \\
\text { these stations may be furnished with reclining chairs or } \\
\text { beds. } \\
\text { Located in a common area so as to enable nursing staff to } \\
\text { simultaneously monitor several patients. } \\
\text { Electricity generator, access to oxygen, and possibility of } \\
\text { monitoring where the nature of the procedures so requires. }\end{array}$ \\
\hline RESTROOMS & $\begin{array}{l}\text { For patients and those accompanying them. } \\
\text { If the ADH is located in the vicinity of the } \\
\text { waiting room, the two spaces may use the } \\
\text { same restrooms. }\end{array}$ & $\begin{array}{l}\text { Sink and toilet. } \\
\text { The number of restrooms required will depend on patient } \\
\text { volumes. }\end{array}$ \\
\hline $\begin{array}{l}\text { STATION FOR } \\
\text { CARE PROVIDERS }\end{array}$ & $\begin{array}{l}\text { Monitoring of patients during their procedures. } \\
\text { Preparation of materials to be used during } \\
\text { procedures. } \\
\text { Preparation of drugs for administration. } \\
\text { Classification of waste products. }\end{array}$ & $\begin{array}{l}\text { Located in a central area or, alternatively, one with full } \\
\text { visibility. } \\
\text { Work station and applications for patient management. }\end{array}$ \\
\hline
\end{tabular}


the general department's administrative staff, thus eliminating the need for administrative personnel devoted exclusively to the $\mathrm{ADH}(86.27 \%$, majority without consensus). However, $\mathrm{ADHs}$ that are separated from the rest of the allergy department will require their own staff.

v. Other support staff. It is necessary to have support available from other auxiliary staff; these auxiliary care professionals are necessary in the event a patient needs to be transferred to another unit or ward after developing an allergic reaction during the procedure. $92.18 \%$ of the allergists surveyed believe that having access to the services of this support staff is recommendable, as these employees may be called on if the need for urgent patient transport arises (consensus).

f) Care quality

The quality of care delivery - understood as the success of procedures and the satisfaction of patients seen in the unitshould be subjected to review. For this purpose, the following should be available, among others:

- Anonymous patient-satisfaction surveys.

- Care-activity indicators.

- Quality indicators.

- Performance indicators.

\subsubsection{Area-by-area description of an ADH}

See Table 3, Table 4

\section{Requirements for procedures specific to allergology}

In allergy practice, many diagnostic and therapy procedures involve administering allergens to allergic patients. As a result - and notwithstanding the fact that specially trained nursing staff often carry out these tasks-supervision of an allergy specialist is essential, as this physician not only determines the indication of the treatment, but also assesses the clinical outcome of the procedure.

Collaboration between the specialist physician and nursing staff is vital to both the design of SOPs as well as when performing the procedures themselves.

When establishing the required procedure times, we have not only taken into account the times involved to complete the procedure, but also the time devoted to preparation, observation, resolution of complications, education, and data recording (98.8\%, consensus).

Patients of all ages are seen in allergology, from small children to elderly patients. For procedures performed in children, we have factored in both the space requirements and the need for additional time to complete the procedures.

\subsection{Group A procedures}

\subsubsection{A-1. Intraepidermal tests}

\subsubsection{General conditions}

- Testing site: Nursing or diagnostic procedures area (testing room), whether in a hospital facility or in another properly outfitted facility.
Table 4.

\begin{tabular}{|c|c|}
\hline \multicolumn{2}{|c|}{ List of equipment, broken down by space } \\
\hline Space & Equipment \\
\hline Waiting room & $\begin{array}{l}\text { Benches or chairs } \\
\text { Pleasant decoration } \\
\text { Cold water drinking fountain } \\
\text { Entertainment options }\end{array}$ \\
\hline Restrooms & $\begin{array}{l}\text { Toilet-paper dispensers } \\
\text { Paper-towel dispensers } \\
\text { Toilet brushes } \\
\text { Mirrors } \\
\text { Waste-paper basket } \\
\text { Soap dispenser } \\
\text { Clothing hooks } \\
\text { Air freshener }\end{array}$ \\
\hline Reception desk & $\begin{array}{l}\text { Work station: computers, filing cabinets } \\
\text { Printer } \\
\text { Desk } \\
\text { Drawers } \\
\text { Bookshelves or closet } \\
\text { Ergonomic rolling chair } \\
\text { Telephone and fax machine }\end{array}$ \\
\hline Examination room & $\begin{array}{l}\text { Desk with computer and drawers } \\
\text { Rolling chair and chairs for patients } \\
\text { and those accompanying them } \\
\text { Examination table } \\
\text { Appropriate instruments for the type of } \\
\text { examination to be performed } \\
\text { Telephone } \\
\text { Waste-paper basket } \\
\text { Sink with soap dispenser or ethanol- } \\
\text { based hand sanitizer }\end{array}$ \\
\hline $\begin{array}{l}\text { Nursing area/ADH } \\
\text { station }\end{array}$ & $\begin{array}{l}\text { Closet for storage of materials and } \\
\text { medicinal products } \\
\text { Rolling chair or stool with wheels } \\
\text { Instruments required for the procedures } \\
\text { to be carried out } \\
\text { Blood-pressure monitor } \\
\text { Oxygen } \\
\text { Pulse oximeter } \\
\text { Crash cart located in the area or nearby } \\
\text { Gurney for transporting patients if } \\
\text { necessary } \\
\text { Defibrillato } \\
\text { Infusion pump for controlled } \\
\text { administration of intravenous } \\
\text { medication } \\
\text { Monitor with ECG function or } \\
\text { electrocardiograph } \\
\text { Material for preparation of drugs } \\
\text { Refrigerator } \\
\text { Telephone } \\
\text { Direct access to doctors' offices/ } \\
\text { examination rooms }\end{array}$ \\
\hline
\end{tabular}

- Emergency equipment: includes materials for treating anaphylactic reactions in the area where the tests are performed, and with rapid communication lines for contacting emergency-care teams. 
- Other aspects: It is recommended that skin-test results be verified by an allergist; additionally, this allergist shall be easily located should the need for rapid response arise due to allergic reactions during procedures $(90.85 \%)$.

5.1.1.2. Material resources

Intraepidermal tests:

- Standardized allergen extracts, properly stored $\left(2^{\circ}\right.$ to $\left.8^{\circ}\right)$ and in adherence of regulations on expiration monitoring.

- Controls: positive control with histamine hydrochloride $(10 \mathrm{mg} / \mathrm{ml})$ and negative control consisting of the solvent used to prepare the allergens or a $0.9 \%$ saline solution $(\mathrm{ClNa})$

- Standardized, sterile lancets (1 mm).

- Materials for evaluating and archiving test results: manual or digital recording systems, specific templates, skin marker pen, and a millimeter ruler or tool for measuring wheal size.

- Others: latex-free gloves, blotting paper, stopwatch, receptacle for hazardous or biohazardous waste, alcohol, razor for shaving, etc.

5.1.1.3. Staff

- Nurses with specific training.

- Allergy specialist.

\subsubsection{A-2. Intradermal tests}

\subsubsection{Material resources}

- Allergens:

- Hymenoptera venoms

- Drugs: active ingredients and excipients

- Occupational allergens

- Others: rare allergens or new allergens under study

- Controls: positive control with histamine hydrochloride $(0.1 \mathrm{mg} / \mathrm{ml})$ and negative control consisting of the solvent used to prepare the allergens or a $0.9 \%$ saline solution $(\mathrm{ClNa})$.

- U-100 $1 \mathrm{~mL} 28$ gauge $\times 1 / 2$ " needle, with no dead space and permanently attached needle

- Materials for evaluating and archiving test results: methods for recording results (usually a skin marker pen and transparent bandaging), specific templates, and a millimeter ruler or tool for measuring wheal size.

- Others: hydroalcoholic gel, transparent antiseptic, gauze

5.1.2.2. Staff

- Nurses with specific training.

- Allergy specialist.

\subsubsection{Times for A.1 and A.2}

The results of the survey revealed that there was no consensus surrounding the time devoted by nursing staff to intraepidermal and intradermal tests. Most specialists surveyed $(69.93 \%)$ stated that, on average, nurses require 15-20 minutes per patient to prepare, perform, and record the results of prick/ intradermal skin tests (preparation, test performance, and recording of results included; item 47).

The more complex intradermal tests to diagnose drug allergies involve a greater number of agents and dilutions (e.g. general anesthetic drugs, beta-lactam antibiotics, contrast agents for radiological tests). $94.46 \%$ of the specialists surveyed (consensus on item 48) stated that it is recommendable to allot at least 45 minutes of work by a nurse for each patient due to this increased complexity.

\subsubsection{A-3. Epicutaneous or patch tests}

\subsubsection{General conditions}

- Testing site: Nursing or diagnostic procedures area (testing room), whether this be located in a hospital facility or in another properly outfitted location, provided the privacy of patients is safeguarded (these tests are performed on the backside of patients). These tests may be performed in other spaces in the department (for example, in an examination room).

- Material for treating local allergic reactions: usually applied to the part of the body where the test was performed, and requiring simple local treatment (e.g. gauze, topical corticosteroids, and dressing).

- Other aspects: It is recommended that skin-test results be verified by an allergist.

\subsubsection{Material resources}

- Patches with chambers attached to hypoallergenic adhesive material (e.g. Finn Chamber system, Curates, IQ Patch Test).

- Batteries of standard, commercially available patch-test systems.

- Possibility of using entire panels of patch tests.

- Material for application of non-standardized allergen patches (e.g. drugs, foods, latex), such as $0.9 \%$ saline solution $(\mathrm{ClNa})$, vaseline, or other vehicles.

Others: Hypoallergenic adhesive strips, hypoallergenic permanent marker, topical antiseptic cleanser for use before the patch is applied, disposable shaving razor.

\subsubsection{Staff}

- Allergy specialist or nursing staff with specific training.

- Pharmacist with specific training in the preparation of epicutaneous extracts with drugs or excipients.

\subsubsection{Time}

The procedure is performed over 3 working days, though this time may be extended to 5. On the first of these days, the patches are placed. The time required to prepare the material varies according to the complexity of the battery used; this may be as short as 10 minutes when placing a commercial patch-test panel such as the True-Test ${ }^{\mathrm{TM}}$ system (including 4 minutes for the patient to both enter and exit the space) or as long as 20 minutes in the case of complex batteries.

The patches are removed on the second day, and the patient must enter the room on two occasions: once when the patch is lifted off the skin and a second time for the reading of the results. The total estimated time required is 10 minutes.

The third day is used only to take a reading, taking some 10 minutes.

\subsubsection{A-4. Spirometry}

\subsubsection{General conditions}

- Testing site: A calm space devoted specifically to this type of testing, enabling patients to concentrate in surroundings that are distraction-free and do not 
interfere with the test. The space must be of sufficient size so that the patient may be positioned comfortably, taking into account the fact that some patients will need to be maneuvered in a wheelchair. For this reason, the minimum recommended size of this space is $2.5 \times 3 \mathrm{~m}$, with doors measuring $120 \mathrm{~cm}$ in width.

- Material for treating incidents: materials necessary to treat such conditions as respiratory failure (i.e. bronchodilators, holding chambers, pulse oximeter, oxygen source, parenteral corticosteroids). Rapid line of communication with emergency-care services.

- Other aspects: it is recommended that an allergy specialist determine the appropriateness of the procedure and assess the results.

\subsubsection{Material resources}

- Spirometer and instruments used for calibration.

- Scale, stadiometer, and indoor meteorological instrumentation (thermometer-mercury barometerhygrometer to measure relative humidity)

- Rigid mouthpieces, adapters for pediatric patients, disposable filters, noseclips.

- Bronchodilator medication, holding chambers.

- Others: stethoscope, pulse oximeter, bronchodilator medication (i.e. salbutamol, formoterol, ipratropium bromide), and a stopwatch.

\subsubsection{Staff}

Attending physician locatable during procedures.

Nursing staff with a minimum 6-month training in an accredited pulmonary function laboratory and able to perform reproducible maneuvers in accordance with quality standards when guiding patients through the process. These nurses should have performed at least 25 examinations under supervision. Each professional must be well-versed in emergency procedures.

To perform a spirometry test, it is sufficient to have trained nursing staff physically present and an allergy specialist who can be easily located (95.53\%, Delphi consensus).

\subsubsection{Time}

The total time required to perform the procedure, including time devoted by both the patient and nursing staff, is 15 minutes. This comprises data entry, recording of anthropometric parameters, the procedure itself, and any instructions required.

\subsubsection{A-5. Bronchodilator test}

\subsubsection{General conditions}

The same as for simple spirometry.

\subsubsection{Material resources}

Similar to the requirements for spirometry - though with additional bronchodilator agents (i.e. salbutamol, formoterol, ipratropium bromide) - and a stopwatch.

\subsubsection{Staff}

Similar to the requirements for spirometry. To perform a bronchodilator test, it is sufficient to have trained nursing staff physically present and an allergy specialist who can be easily located (94.41\%, Delphi consensus).

\subsubsection{Time}

Patients will need an additional 20 -minute waiting period following the spirometry test, though the test itself only takes an extra 5 minutes. This difference is due to the fact that it is not necessary to take the patient's body measurements (height and weight) or provide them with previous instructions on the procedure.

5.1.5.5. Times to perform A-4 and A-5 tests (adjusted for pediatric patients)

When performing respiratory-function tests in children, an additional $15 \%$ time period should be budgeted with respect to the time required for adult patients (97.39\%, Delphi consensus).

\subsubsection{A-6. Impulse oscillometry}

\subsubsection{General conditions}

Similar to the requirements for spirometry.

\subsubsection{Material resources}

In addition to the material needed to perform a spirometry test, this procedure requires an impulse oscillometry system with a pneumotachometer and airflow and pressure transducer and also a calibration syringe.

\subsubsection{Staff}

Similar to the requirements for spirometry.

\subsubsection{Time}

The total time required to perform the procedure, including the time spent by both the patient and nursing staff, is 20 minutes. This period comprises data entry, recording of anthropometric parameters, the procedure itself, and any instructions required. This does not include the time required to calibrate the oscillometry system.

\subsubsection{A-7. Peak expiratory flow rate}

\subsubsection{General conditions}

- Testing site: This procedure may be conducted in any space that is calm (e.g. examination room, respiratory testing room), as this helps patients focus and eliminates distractions that may interfere with the procedure and the recording of results.

- Other aspects: it is recommended that an allergy specialist determine the appropriateness of the procedure and assess the results.

\subsubsection{Material resources}

Manual meters (some officially recognized models are: Vitalograph, Sibelmed, PF Control, Clement Clark, Mini Wright, Assess, TruZone, and PF Leti).

Electronic meters There are also small electronic meters available on the market, which are outfitted with a computer program. The advantage of such systems is that they eliminate the need to manually record data, offer greater precision, and can log all information in real time.

Paper-based or digital means of recording results.

\subsubsection{Staff}

A registered nurse adequately trained to explain the technique or, alternatively, the physician him or herself. 


\subsubsection{Time}

10 minutes to explain the procedure and show how it is performed, though executing the procedure only takes roughly 3-5 minutes.

\subsubsection{A-8. Measurement of exhaled nitric oxide}

\subsubsection{General conditions}

- Testing site: It is recommendable to measure fractional concentration of exhaled nitric oxide (FeNO) in a soundproof room (to help the patient concentrate on the measurement procedure). The room should have a constant temperature and humidity and be free of substances that may interfere with the test (e.g. volatile anesthetic gases and alcohol-containing disinfectants). Portable models are available, allowing for the procedure to be carried out in an examination room.

- Other aspects: it is recommended that an allergy specialist determine the appropriateness of the procedure and assess the results.

\subsubsection{Material resources}

Materials: Systems that may be used:

- NO-VARIO Analyser (Filt, Berlin, Germany) for nasal, bronchial, and alveolar NO concentrations (electrochemical sensor).

- NIOX-MiNO (Aerocrine, AB, Solna, Sweden) electrochemical analyzer or NIOX-VERO (Aerocrine $\mathrm{AB})$ electrochemic analyzer (currently replacing the NIOX-MINO).

\subsubsection{Staff}

Nursing staff with a minimum 6-month training in an accredited pulmonary function laboratory and with the ability to perform reproducible maneuvers at standards of quality when guiding patients through the process.

\subsubsection{Time}

The total time required to perform the procedure, including the time devoted by both the patient and nursing staff, is 20 minutes. This period comprises data entry and the procedure itself, as well as any instructions required.

\subsubsection{A-9. Nasal endoscopy}

\subsubsection{General conditions}

Testing site: This should be performed in a specific ENT examination room furnished with an appropriate reclining chair

\subsubsection{Material resources}

- Reclining chair fit for examining ENT patients.

- Rhinoscope, forehead lamp, or nasal endoscopy system (rigid or flexible nasal endoscope, light source, camera, screen, image-recording system).

- Saline solution, topical nasal vasoconstrictor drugs, local anesthesia with vasoconstrictor properties.

- Complication-prevention pack (nasal packing materials for epistaxis, bayonet forceps, suction system, silver nitrate cautery sticks)

- Others: vinyl gloves, tissues, gauze, forceps, etc.

\subsubsection{Staff}

A specialist physician with specific training in the procedure, with nursing staff located nearby.

\subsubsection{Time}

Most of the allergists surveyed for this document (78.43\%) consider nasal endoscopy to be a low-complexity procedure, as each patient only requires 15 minutes of the physician's time. The time required of the patient is 20 minutes.

\subsubsection{A-10. Nasal cytology}

\subsubsection{General conditions}

Testing site: This should be performed in a specific ENT examination room furnished with an appropriate reclining chair.

\subsubsection{Material resources}

- Reclining chair fit for examining ENT patients.

- Rhinoscope, forehead lamp.

- Material necessary for collecting and processing samples: cytology brush or curette or system for nasal lavage, microscope slide, cover slips, pipettes, staining and fixing liquids, centrifuge, etc.

- Latex and/or vinyl gloves.

- Complication-prevention pack (nasal packing materials for epistaxis, bayonet forceps, suction system, silver nitrate cautery sticks).

\subsubsection{Staff}

The same requirements that apply to nasal endoscopy.

\subsubsection{Time}

This procedure requires 10 minutes of staff time per patient. The time required of the patient is 15 minutes.

\subsubsection{A-11. Rhinomanometry}

\subsubsection{General conditions}

Similar to those for nasal endoscopy and cytology.

\subsubsection{Material resources}

- Rhinomanomentry measuring device (most commonly used technique: active anterior rhinomanometry) and a computer with the appropriate software installed.

- Meteorological instrumentation to measure atmospheric conditions.

- Armchair for the patient and chair/stool for the person performing the procedure

- System for recording results (paper or digital).

- Others: visual analog scale (with millimeter-length units or using a ruler), topical nasal vasocontrictor medication, and adhesive tape for dilating the ala nasi.

\subsubsection{Staff}

One nurse or specialist physician, with specific training on how to implement this technique.

The staff requirements are the same as for the following: acoustic rhinometry, olfactometry, measurement of peak inspiratory flow rate (PIFR) and nasal nitric oxide.

\subsubsection{Time}

This procedure requires a total of 30 minutes of staff time per patient, including time spent informing patients, conducting 
the procedure, and interpreting the results. As the patient must be present throughout the procedure, they must devote 35 minutes of their time.

\subsubsection{A-12. Acoustic rhinometry}

\subsubsection{General conditions}

Similar to those for nasal endoscopy.

\subsubsection{Material resources}

- Acoustic rhinometer and a computer with the appropriate software installed (acoustic-pulse generator, tube or pipe for transmission of the sound wave, analog-digital conversion system for data processing).

- Armchair for the patient and chair/stool for the nurse.

- Meteorological instrumentation for measuring atmospheric conditions and sound-level meter.

- Nosepieces of different sizes and either gel sealant or vaseline.

- Digital or paper-based system for recording results.

- Visual analog scale (with millimeter-length units or using a ruler)

- Others: Hydroalcoholic gel, latex and/or vinyl gloves, topical nasal vasoconstrictor medication.

\subsubsection{Staff}

One nurse or specialist physician with specific training on how to implement this technique.

\subsubsection{Time}

This procedure requires a total of 30 minutes of staff time per patient, including time spent on informing patients, conducting the procedure, and interpreting the results. As the patient must be present throughout the procedure, they must devote 35 minutes of their time to the process.

\subsubsection{A-13. Olfactometry}

\subsubsection{General conditions}

Testing site: This procedure may be performed in a specific examination room or in a general-use examination room.

\subsubsection{Material resources}

- Validated olfactometry system.

- Meteorological instrumentation for measuring atmospheric conditions.

- Data collection form, visual analog scale.

\subsubsection{Staff}

This procedure can be carried out by a trained nurse (96.73\%, Delphi consensus).

\subsubsection{Time}

This procedure requires a total of 30 minutes of staff time per patient, including time spent on informing patients, conducting the procedure, and interpreting the results. As the patient must be present throughout the procedure, they must devote 35 minutes of their time to the entire process

\subsubsection{A-14. Measurement of peak inspiratory flow rate (PIFR)}

\subsubsection{General conditions}

- Testing site: This procedure may be conducted in any space that is calm (e.g. examination room, respiratory testing room), as this helps patients focus and eliminates distractions that my interfere with the procedure and the recording of results.

- Other aspects: it is recommended that an allergy specialist determine the appropriateness of the procedure and assess the results.

5.1.14.2. Material resources

- System for measuring PIFR.

- Data collection form.

\subsubsection{Staff}

One nurse or specialist physician with specific training.

\subsubsection{Time}

For each patient, it takes a total of 5 minutes to explain and carry out the procedure. As the patient must be present throughout the procedure, they must devote 10 minutes of their time to the entire process.

\subsubsection{A-15. Nasal nitric oxide}

\subsubsection{General conditions}

- Testing site: It is recommendable to measure fractional concentration of exhaled nitric oxide (FeNO) in a soundproof room (to help the patient concentrate on the measurement procedure). The room should have a constant temperature and humidity and be free of substances that may interfere with the test (e.g. volatile anesthetic gases and alcohol-containing disinfectants). Portable models are available, allowing for the procedure to be carried out in an examination room.

- Other aspects: it is recommended that an allergy specialist determine the appropriateness of the procedure and assess the results.

\subsubsection{Material resources}

- Device for measuring nasal nitric oxide.

- Chair for the patient and chair/stool for the nurse.

- Meteorological instrumentation for measuring atmospheric conditions.

- Recording system.

\subsubsection{Staff}

One nurse or specialist physician with specific training.

\subsubsection{Time}

This procedure requires a total of 10 minutes of staff time per patient, including time spent on informing patients and explaining and conducting the procedure. As the patient must be present throughout the procedure, they must devote 15 minutes of their time to the process.

\subsubsection{A-16. Specific conjunctival provocation}

\subsubsection{General conditions}

- Testing site: Nursing or diagnostic procedures area (testing room), whether this be located in a hospital facility or in another properly outfitted center.

- Emergency equipment: Although systemic complications are rare, the unit should have materials to treat anaphylaxis and have a system in place for rapid 
communication with emergency-care services. It is recommendable to have a spirometer nearby.

- Other aspects: it is recommended that an allergy specialist determine the appropriateness of conjunctival provocation and assess the test results.

5.1.16.2. Material resources

- Standardized allergen extracts for use in ocular provocation testing

- Diluents, if the extracts are lyophilized and must be reconstituted

- Diluent or saline solution as a negative control

- Syringes, pipettes where necessary (where extracts are not available in drip-administration receptacles)

- Form for recording symptoms. Ruler for visual analog scale

- Access to a spirometer

- Topical ophthalmic vasoconstrictor, corticosteroids (oral, topical, parenteral).

\subsubsection{Staff}

Conjunctival provocation testing with allergen extracts may be performed by a trained nurse provided there is an allergist nearby (97.39\%, Delphi consensus).

\subsubsection{Time}

This procedure requires the physician to devote 5-10 minutes of their time per patient. Nursing staff require a total of between 90 and 120 minutes to prepare the extracts and conduct the test. The procedure requires approximately 120 minutes of the patient's time.

\subsubsection{A-17. Tests of physical urticaria}

\subsubsection{General conditions}

- Testing site: Nursing or diagnostic procedures area (testing room), whether this be located in a hospital facility or in another properly outfitted center.

- Emergency equipment: Although systemic complications are rare, the unit should have materials to treat anaphylaxis.

- Other aspects: it is recommended that an allergy specialist determine the appropriateness of physical urticaria testing and assess the test results.

\subsubsection{Material resources}

As per published protocols, when diagnosing physical (inducible) urticarias, it is recommended to use specific devices and materials, such as ice cubes, so the procedure may be performed in the most suitable manner (97.39\%, Delphi consensus).

\subsubsection{Staff}

One nurse or specialist physician with specific training. The physician must remain nearby while the procedure is being performed.

\subsubsection{7-1. A-17.1. Aquagenic urticaria}

\subsubsection{7-1.1. Material resources}

- Thermometer with which to measure water temperature.

- Watch or stopwatch.
- Others: cloth or compress for moistening, water, receptacles for water.

\subsubsection{7-1.2. Time}

For this test, patients need to allot approximately 60 minutes in total; this time is broken down as follows:

- Preparation of the patient and test materials: 2-5 minutes.

- Applying the moist compress: 30 minutes.

- Reading of results: 2-30 minutes.

\subsubsection{7-2. A.17.2. Dermographism/urticaria factitia}

\subsubsection{7-2.1. Material resources}

Two specific instruments may be used to test for this condition:

- Calibrated dermographometer: Pen-shaped model distributed by HTZ Limited (Vulcan Way, New Addington, Croydon, Surrey, UK) http:/www.htz.biz/ dermagraphic_tester.htm

- Fric-test ${ }^{\circledR}$ (Biomedizinische Werkstätte de Charite, Berlin)

In the absence of the aforementioned systems, a blunt object such as a pen cap or depressor may be used.

\subsubsection{7-2.2. Time}

Tests for diagnosing dermographism/urticaria factitia require 10 minutes of time from nursing staff per patient ( $86.93 \%$ of specialists surveyed, though without consensus).

\subsubsection{7-3. A-17.3. Heat-induced urticaria}

\subsubsection{7-3.1. Material resources}

- Heat source: microwave or portable/camping stove.

- Metal or glass receptacle for drinking.

- The liquid to be heated (water).

- Thermometer to measure water temperature.

- Watch or stopwatch.

\subsubsection{7-3.2. Time}

For this test, patients need to allot approximately 20 minutes in total; this time is broken down as follows:

- Material preparation by nursing staff: 2-5 minutes.

- Application of heat source: 5 minutes

- Reading of results: 10 minutes.

Tests for diagnosing heat-induced urticaria require 10 minutes of time from nursing staff per patient $(84.97 \%$ of specialists surveyed, though without consensus).

Note: the TempTest ${ }^{\circledR} 4.0$ (Moxie Gmbh) system may be used in lieu of a classical heat source (see A.17.8 below).

\subsubsection{7-4. A-17.4. Procedure for pressure urticaria}

\subsubsection{7-4.1. Material resources}

Two different procedures may be used:

- Two 7-kg weights (in total) and a 3-cm-wide belt. - cylinders measuring $1.5 \mathrm{~cm}$ in diameter and weighing $2.28 \mathrm{~kg}(127 \mathrm{kPa})$ to $4.79 \mathrm{~kg}(266 \mathrm{kPa})$

- cylinder measuring $1.5 \mathrm{~cm}$ in diameter and weighing $2.5 \mathrm{~kg}(139 \mathrm{kPa})$ to $3.5 \mathrm{~kg}(194 \mathrm{kPa})$

- cylinders measuring $6.5 \mathrm{~cm}$ in diameter and weighing $5 \mathrm{~kg}(14.8 \mathrm{kPa})$

- support for cylinders 
- Using a pen-shaped calibrated dermographometer with a $0.9 \mathrm{~mm}$ point at $100 \mathrm{~g} / \mathrm{mm}^{2}$ (Dermagraphic tester, HTZ Limited).

\subsubsection{7-4.2. Time}

For this test, patients need to allot 5 to 20 minutes in total; this time is broken down as follows:

- Patient identification and preparation: 2-5 minutes (upper limit)

- Test performance:

- With weights or metal cylinders: 15-20 minutes

- With a dermographometer: 70 seconds.

The test results are read immediately after the procedure and at 30 minutes, with an accelerated reading at 6 hours. Follow-up is required 24 hours later in some cases to determine the presence of delayed-onset reactions.

Tests for diagnosing pressure urticaria require 70 seconds of time from nursing staff when a dermographometer is used and 5 minutes per patient when weights or cylinders are used ( $82.35 \%$ of specialists surveyed, though without consensus).

\subsubsection{7-5. A-17.5. Solar urticaria. Phototesting/ photoprovocation}

\subsubsection{7-5.1. Specific material resources}

Artificial light source: PUVA lamp (MED UVA, 3-16 J/. $\mathrm{cm}^{2}$ ), UVB 6-108 $\mathrm{mJ} / \mathrm{cm}^{2}$ or solar-type fluorescent tubes

\subsubsection{7-5.2. Time}

To conduct phototesting/protoprovocation for solar urticaria, the average time required of nursing staff is 30 60 minutes per patient $(91.50 \%$, consensus $)$; this is also the same time commitment required of patients undergoing the procedure.

\subsubsection{7-6. A-17.6. Autologous serum skin test}

\subsubsection{7-6.1. Material resources}

- To draw a venous blood sample from the patient:

- Informational sticker, specific to each center (patient identification).

- Hydroalcoholic gel.

- Vacuum tube for biochemical testing, 8.5-10 ml, with gel (yellow/red top) WITHOUT ADDITIVES.

- Extraction system: sterile Vacutainer ${ }^{\circledR}$-type system for drawing blood, with safety mechanism (wings); alternatively, IV needles.

- Compressor.

- Sterile gauze.

- Transparent topical antiseptic.

- Sample preparation:

- Centrifuge

- Centrifuge tube rack for previously centrifuged serum

- Inoculation of patient serum (intradermal):

- U-100 1-ml syringe, with no dead space and a blunt, 18 -gauge $\times 1 \frac{1}{2}$ ” needle to collect autologous serum from the patient.

- U-100 1-ml syringes, with no dead space and a 29 -gauge $\times 1 / 2$ " attached needle for intradermal inoculation.

- Sterile gauze.
- Transparent topical antiseptic.

- Receptacle for disposal of hazardous or biohazardous waste.

- Hypoallergenic permanent marker.

- Negative control: Sodium chloride $0.9 \%$.

- Positive control: Histamine hydrochloride $(0.1 \mathrm{mg} / \mathrm{ml})$.

- Standard lancet for prick testing.

- Blotting paper or cellulose napkin.

- Watch or stopwatch.

- Transparent microperforated tape.

\subsubsection{7-6.2. Time}

The time spent by the patient is approximately 120 minutes, distributed as follows:

- Drawing of blood sample: Patient identification, preparation, and drawing of blood: 5 minutes.

- Sample preparation: Time for clotting: 30 minutes; centrifugation: 15 minutes.

- Inoculation of patient serum (intradermal): Patient identification, preparation and inoculation of serum and controls: 15 minutes.

- Reading and interpretation of test results at 15, 30, and 60 minutes.

Nursing staff devote 20-25 minutes to this test.

\subsubsection{7-7. A-17.7. Cholinergic urticaria}

\subsubsection{7-7.1. Material resources}

- Treadmill/stationary bike

- Blood-pressure monitor and pulse oximeter.

- Scale and stadiometer

\subsubsection{7-7.2. Time}

Physical exercise until perspiration (5-10 minutes). Following this, an additional 15 minutes following initiation of perspiration.

The average time required for the test to diagnose cholinergic urticaria is 30 minutes per patient $(91.50 \%$, consensus).

In case of doubt between cholinergic urticaria and exerciseinduced anaphylaxis, exercise-induced anaphylaxis must be ruled out with a hot-water immersion test, waiting at least 48 hours after the treadmill test due to the existence of a refractory period.

\subsubsection{7-7.3. Hot-water immersion test}

\subsubsection{7-7.4. Material/space resources \\ - Basin/Tub \\ - Hot water \\ - Thermometer}

\subsubsection{7-7.5. Time}

As of the time the patient is immersed, the time necessary for their body temperature to raise by $1^{\circ} \mathrm{C}$, followed by an additional 15 minutes.

Total time: 20-25 minutes.

\subsubsection{7-8. A-17.8. Cold-induced urticaria}

There are several variants:

- Ice cube test. 


\subsubsection{7-8.1. Material resources}

- Freezer with ice cubes.

- Latex-free glove or plastic bag where the ice cube is placed.

- Bandage to hold the ice cube against the patient's forearm (optional).

- Watch or stopwatch.

\subsubsection{7-8.2. Time}

- Preparation of the ice cube and other material by nursing staff: 2-5 minutes.

- Duration of ice application: from 5 to 10 minutes, with gradual increases following negative results.

- Reading of results: 10 minutes.

If the test is performed without detection of stimulation thresholds, the test lasts about 15 minutes. If the test lasts for 10 minutes, total time is 20 minutes.

If, in an ideal case, the test is performed with detection of stimulation thresholds through gradual stimulation, the test may take as long as 105-110 minutes.

TempTest ${ }^{\circledR} 4.0$ (Moxie Gmbh).

\subsubsection{7-8.3. Material resources}

- TempTest.

- Watch or stopwatch.

- Transparent plastic film used to mark the test results.

\subsubsection{7-8.4. Time}

The total time required to perform the test is $15-20$ minutes.

- Preparation of the TempTest system and other material by nursing staff: $2-5$ minutes.

- Time of application: 5 minutes.

- Reading of results: 10 minutes.

\subsubsection{A-18. Latex handling test}

\subsubsection{General conditions}

Similar to those that apply when diagnosing physical urticaria.

\subsubsection{Material resources}

- Box of powdered latex gloves to be used during the test

- Box of vinyl or nitrile gloves for use by the care professional performing the test.

- Receptacle, water, gauzes for moistening, disposable tongue depressor

- Watch or stopwatch.

- Device for measuring peak flow or spirometer to measure respiratory flow.

\subsubsection{Staff}

Nursing staff specifically trained in conducting the test and an allergy specialist to assess possible allergic reactions.

\subsubsection{Time}

The time the patient may need to complete the test may vary based on the protocol used (e.g. skin, mucosal, or both skin and mucosal), and as a result this test may take between 30 and 60 minutes. If the study is first performed with a finger cot and then with the entire hand, the entire procedure may take up to 120 minutes

The time devoted to the test by staff is 10 minutes

\subsubsection{A-19. Subcutaneous and sublingual conventional immunotherapy}

\subsubsection{General conditions}

- Testing site: Nursing area or space especially devoted to allergy testing. Immunotherapy must always be performed in a health center.

- Emergency equipment: includes materials necessary to treat anaphylactic reactions, and with possibility of rapid communication with emergency-care teams.

- Other aspects: The unit should have the features listed below.

- A protocol for the administration of allergen immunotherapy (AIT).

- A patient file containing the clinical report and a record of treatments administered with incidents.

- Coordination with other health facilities and the primary-care system in the event patients are referred for AIT administration.

- An action protocol in the event of adverse reactions.

- In case of serious reactions or reactions related to new treatments (recently released on the market or having a newly modified composition or concentration), a means of recording and notifying these reactions to pharmacovigilance teams.

\subsubsection{Material resources}

- A specific doctor's office/examination room or a specially designated nursing area, such as an area where patients are examined and assessed within an examination room. However, these spaces may be shared and used for other activities.

- 1-ml syringes with milliliter scale markings with needles for subcutaneous injection.

- Instruments for measuring vital signs (i.e. heart rate, blood pressure, oxygen saturation), peak flow, spirometer, stethoscope, and blood-pressure meter.

- Others: ruler for measurement of local reactions, ice, syringes for intramuscular administration, injection systems, fluids (sodium chloride $0.9 \%$ saline solution), cotton/gauze, antiseptics (chlorhexidine or alcohol), timers, tourniquets.

- Oral, parenteral, and topical medication for treatment of local reactions: epinephrine, antihistamines, corticosteroids, and short-acting beta agonists.

- Telephone for communicating with and alerting emergency-care teams.

\subsubsection{Staff}

Nursing staff or specialist physician with specific training in immunotherapy administration.

The specialist shall be responsible for determining the appropriateness of the treatment, assessing possible allergic reactions, and making any treatment modifications.

\subsubsection{Time}

Patients will need to devote a total of 40 minutes for every dose administered, as the process requires a 30 -minute observation period.

The time devoted to the test by staff is approximately 10 minutes per patient, including the recording of the 
dose and review of how well the patient tolerated the previous dose.

\subsubsection{A-20. Parenteral self-administration of treatment}

Definition:Self-administration is subcutaneous or intravenous administration of treatment by the patient themselves or their carers outside of a health facility.

The aim of self-administration is to save time and resources, lower absenteeism, and allow patients to live autonomously and at a high quality of life.

In-home parenteral treatment began over 40 years ago and was first practiced by hemophilia patients. Reports from other in-home treatment programs show that self-administration leads to increased patient confidence, knowledge of their disease, and improvements in quality of life and satisfaction.

The first data on self-administered $\mathrm{C} 1$ esterase inhibitor concentrates for episodes of hereditary angioedema (HAE) in the European Union date from 1989. Information on this initiative reveal that the benefits of self-administration include lower frequency and intensity of episodes, improved quality of life, and decreased absenteeism.

Teaching methods for self-administration of parenteral treatment can be applied to other products, such as epinephrine. The self-administration program for drug treatment should be adapted to the way the drug is presented and the characteristics of the substance.

Methodology: individual intervention using skill-building techniques.

These techniques are eminently useful in helping patients acquire specific skills and develop their ability to act, teaching them how to respond to real-life situations and implement previously decided changes. Use of role-playing, situation analysis, and real-life practices.

Population: Patients diagnosed with HAE or anaphylaxis as well as their primary caregivers or individuals accompanying them. The patients who take part are those who both require treatment and meet the inclusion criteria, e.g. medical indication, motivation, biopsychosocial conditions, treatment compliance.

Number of sessions: Varies based on the content to be covered and the pace of learning. It is recommended to hold 1 to 7 sessions.

Session duration: It is recommended that each session last between 30 and 120 minutes depending on the efficiency of each meeting. For sessions lasting more than 60 minutes, a break will be necessary to keep participants' concentration levels up.

Frequency: Determined by the topic to be covered and the characteristics of the attendees. It is advisable for there to be a program starting every week. Periodic refresher sessions.

Scheduling: It is recommended to schedule the sessions far enough in advance in order to allow participants to plan accordingly.

Site: It is recommendable to hold these sessions in the same place throughout the process. The space should offer access to a restroom, be well-lit, have an appropriately sized desk, be used exclusively for educational purposes, and be free from interruption.
Number of participants: One-on-one intervention. At times, the patient and their primary caregiver attend the sessions at the same time.

\subsubsection{Resources used}

- Staff: nurse to lead the workshop.

Materials and documentation: Texts, posters, pamphlets, photographs, slides, videos, etc. Materials required for hand hygiene, for drug self-administration, and to dispose of waste. Certificate accrediting capacity for self-administration.

\subsubsection{Patient/Caregiver commitment}

- Refrain from using the techniques learned in the sessions on others

- Act responsibly and in adherence of asepsis procedures

- Comply with recommended medical oversight

- Conserve the drug in good condition

- Use the concentrate early, with the prescribed quantities, and at the times indicated; if no improvement is seen, report to the emergency room or allergy department

- Fill out the data collection forms and take them to the allergy department so that further prescriptions may be made

- Collect waste material and dispose of it properly

* Failure to comply with these rules may be grounds for temporary or permanent discontinuation of this treatment modality.

\subsubsection{Assessment form}

Once the training program has been completed, participants must be tested to ensure that they have understood and can carry out each of the steps covered.

\subsubsection{A-21. Patient education}

Patient education is an educational process and publichealth initiative whose primary objective is to qualitatively improve people's health, allowing individuals increased control over their health, empowering them to improve their state, and enabling the citizenry to take greater responsibility in defending their own health and that of others.

Patient education is rooted in two basic rights: the right to education and the right to health. Both of these rights are fully recognized in the Spanish constitution of 1978 (articles 27 and 43, respectively).

The term therapeutic patient education was first recognized by the World Health Organization (WHO) Regional Office for Europe in 1998. According to the WHO, therapeutic patient education is an integral and continuing part of patient care and one that is centered on the patient. The aim is to help patients acquire or maintain the skills they need to better manage their lives with the disease.

It comprises activities related to the patient's disease and the treatment prescribed for the disease, including awarenessraising, information, learning, and psychosocial support. This requires planning, methodology, and continuity, and as a result cannot accommodate improvisation or one-off actions.

Objective: To broaden the population's knowledge of health issues and develop values, attitudes, and personal skills conducive to good health.

The methodology used in patient education must be understood as the means of transmitting the message, and 
this methodology must be active and participatory. The aims are as follows:

- Identify patients' educational needs, priorities, and motivation so that they may set viable short-term goals and increase their confidence in their ability to effect changes.

- Offer information that is accessible and appropriate, enabling patients to attain a correct level of self care and responsibility with regard to their disease and the treatment thereof.

- Promote healthy lifestyle habits that may improve the state of their health.

- Take preventive measures to prevent disease and/or assuage its adverse aspects.

- Assess the information provided, the extent to which it has been understood, the outcomes of this learning, and the impact of the program.

Patient education should exist in tandem with all therapy procedures.

\subsubsection{Physical space}

A room is required for patient education. This may or may not be located on the premises of the allergy department or service.

\subsubsection{Material resources}

An audiovisual projection system.

Drug placebos (e.g. different types of inhalers, selfinjection systems, nasal sprays) and demonstration devices (peak expiratory flow, etc.).

\subsubsection{Staff}

Medical professional (physician/registered nurse) with appropriate training.

\subsubsection{Time}

The duration of patient-education courses depend on the material covered. On average, sessions should last from 15 to 30 minutes.

\subsection{Group $B$ and $C$ procedures}

Procedures normally falling under Group A which, due to the particular circumstances of the patient or the disease, become classified as Group B or C, are not given repeat description here, as the methodology and materials used are the same, and the only difference is that the procedures are performed in an $\mathrm{ADH}$. Emergency equipment is common to all procedures and forms part of the basic equipment of an $\mathrm{ADH}$, and therefore is not detailed further in this section.

\subsubsection{BC-1 Nonspecific bronchial challenge}

Nonspecific bronchial challenge may be performed with various agents (direct or indirect stimuli). The aim of this document is not to precisely describe each of these; rather, an overall assessment of these challenges is offered in terms of the resources and time they require.

\subsubsection{Physical space}

This may be the same space used for spirometry and other respiratory-function tests.

\subsubsection{Material resources}

Similar to those required for the bronchodilator test, with the addition of:

- Bronchoconstrictor agent (methacholine, mannitol, adenosine)

- Nebulizer/Dosimeter

- Saline solution or the excipient used as a diluent in bronchial challenge testing.

\subsubsection{Staff}

A nonspecific bronchial challenge may be performed by nursing staff, though these professionals must be trained in carrying out respiratory function tests (as described for spirometry). It is recommended that these staff members not be asthmatic themselves. Tests must be supervised by an appropriately trained allergy specialist present during the procedure. This individual will be the utmost authority figure while the test is being performed and, if necessary, will direct any therapy maneuvers necessary to deactivate any incidents and return the patient to the state they were in prior to the test.

\subsubsection{Time}

To screen for nonspecific bronchial hyperresponsiveness (methacholine or adenosine) with a dosimeter, it is recommended to allot 45 minutes per patient $(90.20 \%$, consensus).

To screen for nonspecific bronchial hyperresponsiveness (methacholine or adenosine) using the tidal volume method, it is recommended to allot 60 minutes per patient $(94.12 \%$, consensus).

\subsubsection{BC-2. Exercise test}

\subsubsection{Physical space}

Similar to the requirements for spirometry, but with the additional requirement of a space for a treadmill or ergonomic bicycle.

\subsubsection{Material resources}

Similar to the requirements for the bronchodilator test, plus a treadmill or ergonomic bicycle.

\subsubsection{Staff}

Similar to those that apply to nonspecific bronchial challenge.

\subsubsection{Time}

The total time required to perform the test is 30 minutes; during this time, patients must remain under observation, and as a result the staff-time requirement is the same.

\subsubsection{BC-3. Specific bronchial challenge (SBC)}

\subsubsection{Physical space}

This test requires an enclosed challenge room equipped with an adequate exhaust-ventilation system or closedcircuit device in order to prevent inadvertent exposure of the technicians and the patient after the challenge has concluded.

\subsubsection{Material resources}

During a specific bronchial challenge, patients should wear protective overalls, caps, eye protection, shoes, and non-latex gloves to avoid, as far as possible, direct skin exposure to the tested agent. 


\subsubsection{Staff}

An allergy specialist with adequate training and a nurse with specific training and experience in performing this test.

\subsubsection{Time}

Specific bronchial challenges vary in length based on the number of doses to be administered and the time it takes to provoke a positive result, at which point the test is concluded. On average, the test takes 120 minutes, after which the patient must remain under observation, possibly adding another 120 minutes to the procedure length. In certain cases, patients require monitoring for hours after the procedure has been discontinued.

\subsubsection{BC-4. Induced sputum study}

\subsubsection{Physical space}

A calm area sectioned off from the spaces used for other procedures. The test must be performed in a well-ventilated area so as to prevent excessive concentrations of hypertonic saline solution caused by nebulizer use. During nebulization and coughing/expectoration, medical staff must remain at a distance of no less than 1 meter from the patient, though with the patient in their sight at all times in case an intervention is necessary.

\subsubsection{Material resources}

- Ultrasonic nebulizer (De Vilbiss ${ }^{\circledR}$, Ultra-Neb ${ }^{\circledR} 2000$, Omron ${ }^{\circledR}$, NE U07).

- Spirometer and calibration equipment (meteorological instrumentation) and 3-liter syringe for daily calibration.

- Though not essential, it is recommended to have a pulse oximeter to monitor oxygen saturation if the need arises.

- There must be oxygen-supply equipment in the room where the procedure is performed.

- CPR equipment or trolley.

- Stopwatch or watch (indicating minutes).

- Refrigerator for storage of saline solution and samples.

$-0.9 \%$ isotonic saline solution.

$-3.4 \%$ and $5 \%$ hypertonic saline solution.

- Inhaler with $\beta 2$ adrenergic receptor agonist (salbutamol) and holding chamber.

- 10-ml syringes.

- Sputum-collection containers.

- Noseclip for spirometry.

- Latex-free gloves.

- Disposable cups and water for mouth rinsing.

- Box of tissue paper.

- Data collection form.

\subsubsection{Staff}

A nurse with experience in conducting the procedure and a specifically trained allergy specialist.

\subsubsection{Time}

The total time required is 20 minutes.

\subsubsection{BC-5. Drug provocation test}

\subsubsection{Material resources}

In addition to the facilities of the $\mathrm{ADH}$ proper, it is necessary to have the drugs used in the study, properly split into the dose levels indicated in the provocation-test protocols.

\subsubsection{Staff}

- Nursing staff with experience in carrying out the test.

- Allergy specialist with specific training.

\subsubsection{Time}

On average, the specialist physician must devote 45-60 minutes per patient and per procedure $(90.20 \%$, consensus), and the time required of nursing staff is 60 minutes per patient and procedure ( $90.85 \%$, consensus). Despite this, patients may have to devote up to 5 or 6 hours to the process depending on the drug to be administered, the number of doses, and the expected type of clinical manifestations. Additionally, patients require an additional 2 hours of observation time after the provocation test has concluded ( $94.77 \%$, consensus).

No consensus was reached among the surveyed specialists regarding cases deemed to be of low risk, though most of those surveyed estimate that the procedure requires some 15 minutes from the physician $(86.93 \%)$ and also from the nurse ( $81.70 \%$, majority without consensus). The lack of agreement observed in these cases may stem from the fact that the time required should be equal regardless of the risk level determined by some allergists.

\subsubsection{BC-6. Desensitization to medicinal products}

\subsubsection{Material resources}

In addition to the facilities of the ADH proper, it is necessary to have the drugs used in the study, properly split into the dose levels indicated in the desensitization protocols.

\subsubsection{Staff \\ - Nursing staff with experience in carrying out the test. \\ - Allergy specialist with specific training. \\ - Nursing assistant, administrative staff, and orderly.}

\subsubsection{Time}

For high-risk or high-complexity parenteral desensitization procedures, each member of nursing staff must devote 120 minutes of their time per patient $(92.16 \%$, consensus), though this period may last over 4 or 5 hours in some cases.

\subsubsection{BC-7. Food challenge test}

\subsubsection{Material resources}

\subsubsection{Physical space: Room for food preparation}

Area devoted to the preparation of foods, placebos, masking/blinding, drugs, and dilutions. The space should be furnished with tables where the foods to be consumed by patients are to be placed. Foods should be kept separate from each other to avoid the risk of cross-contamination. Also, foods should be kept out of reach of patients.

- Microwave

- Blender

- Closet for storage of consumables located in the area

- Trays

- High-precision scale

\subsubsection{Staff}

- Adequately trained allergy specialist

- Registered nurse with experience in carrying out the test 


\subsubsection{Time}

On average, the allergy specialist must devote 45-60 minutes per patient $(90.20 \%$, consensus), and the time required of nursing staff is 60 minutes per patient $(90.85 \%$, consensus $)$. Despite this, patients may have to devote up to 5 or 6 hours to the process depending on the food used in the challenge and the number of doses. Additionally, patients require an additional 2 hours of observation time after the provocation test has concluded ( $94.77 \%$, consensus).

No consensus was reached among the surveyed specialists regarding cases deemed to be of low risk, though most of those surveyed estimate that the procedure requires some 15 minutes from the physician $(86.93 \%)$ and also from the nurse $(81.70 \%$, majority without consensus). The lack of agreement observed in these cases may stem from the fact that an equal amount of time is required regardless of the risk level determined by some allergists.

\subsubsection{BC-8. Oral immunotherapy/OIT}

\subsubsection{Physical space: Room for food preparation}

Area devoted to the preparation of solid foods, placebos, masking/blinding, drugs, and dilutions. The space should be furnished with tables where the foods to be consumed by patients are to be placed. Foods should be kept separate from each other to avoid the risk of cross-contamination. Also, foods should be kept out of reach of patients.

- Microwave

- Blender

- Closet for consumables

\subsubsection{Staff}

- Nursing staff with experience in carrying out the test.

- Allergy specialist with specific training.

- Nursing assistant, administrative staff, and orderly.

\subsubsection{Times required for the procedure}

No consensus was reached concerning the required time devoted to these procedures by care professionals. $88.89 \%$ of those surveyed considered that, per day, each nurse assigned to oral desensitization procedures should devote no less than 90 minutes per patient and per challenged food.

The time required of the allergist is less, at about 45 minutes per patient.

\subsubsection{BC-9. Administration of biological drugs}

\subsubsection{Physical space}

Not required.

\subsubsection{Material resources}

In addition to the standard materials required in an $\mathrm{ADH}$, this procedure requires the unit to have the drug to be administered, which shall be properly labeled and stored in optimal conditions.

\subsubsection{Staff}

One nurse, and an allergy specialist locatable at all times.

\subsubsection{Time}

Administration usually takes 10 minutes, though the patient must remain in the facility for an additional 30 minutes after the procedure has concluded.

\subsubsection{BC-10. Immunotherapy}

\subsubsection{Physical space}

No additional physical space required beyond that of the $\mathrm{ADH}$.

5.2.10.2. Staff

- Nursing staff with specific training in immunotherapy administration.

- Specialist physician in allergology to assess possible allergic reactions, make any necessary modifications to the protocol, and indicate treatment for patients, where applicable.

\subsubsection{Time}

As administration of the drug must be followed by a 30 -minute observation period, this treatment requires 40 minutes of the patient's time per dose.

The time devoted to the test by staff is approximately 10 minutes per patient, including the recording of the dose and review of how well the patient tolerated the previous dose.

\subsubsection{BC-11. Hymenoptera-sting challenge}

\subsubsection{Physical space}

This challenge shall be carried out in the $\mathrm{ADH}$ facility, though it is recommended to use an independent space/ enclosed cubicle in the event the hymenenoptera specimens escape.

\subsubsection{Specific material}

- Insects with which to perform the procedure

- Transparent plastic receptacle

$-\mathrm{CO}_{2} \operatorname{tank}$

- Entomology scissors and forceps

\subsubsection{Staff}

Controlled sting challenges may be performed by nursing staff, though an allergy specialist or the attending allergist must be physically present $(94.12 \%)$.

\subsubsection{Time}

The procedure requires around 150 minutes of time per patient from both the allergy specialist and nurse. Performing more than 5 sting challenges in a single workday is not recommended.

Monitoring of heart rate, blood pressure, oxygen saturation, and peak flow for 120 minutes after the test has been conducted. Wheal size should be measured quantitatively 15 minutes after the procedure. If the patient has experienced an anaphylactic reaction, it is recommended that they undergo observation for 6 hours; this observation period may be even longer in cases with refractory systems or airway involvement.

\section{References}

- Agency for Healthcare Research and Quality (AHRQ). Making Health Care Safer: A Critical Analysis of Patient Safety Practices. AHRQ. July, 2001. Available at: http://archive.ahrq. gov/clinic/tp/ptsaftp.htm

- Antolín Amérigo D. Plan Estratégico de Calidad y Seguridad de la SEAIC 2015-2018. Madrid: SEAIC; 2015. Retrieved from: 
http://www.seaic.org/wp-content/plugins/download-monitor/ download.php?id=PE_SEAIC_seguridad_calidad_2015-18. pdf

- Cometto C, Gomez P, Dal Sasso G, Zarate R, de Bortoli S, Falconi C. Enfermería y Seguridad de los Pacientes. Washington, DC. Organización Panamericana de la Salud. 2011.

- De las Marinas MD, García BE, Alamar R, Pérez-Francés C, Martorell C, Hernández D, Análisis de la seguridad clínica de las unidades de Alergología de la Comunidad Valenciana. An Sist Sanit Navar 2017;40:269.78.

- European Academy of Allergology and Clinical Immunology Advocacy Manifesto. http://www.eaaci.org/documents/ EAACI_Advocacy_Manifesto.pdf

- European Commission. DG Health and Consumer Protection. Patient safety - Making it Happen! Luxembourg Declaration on Patient Safety, 5 April 2005. Available at: http://ec.europa. eu/health/ph_overview/Documents/ev_20050405_rd01_ en.pdf

- European Union Network for Patient Safety and Quality of Care. PaSQ; 2011. Available at: http://www.pasq.eu

- Joint Commission 2012. Improving patient and worker safety. Opportunities for synergy, collaboration and innovation. Available at: http://www.jointcommission.org/assets/1/18/tjcimprovingpatientandworkersafety-monograph.pdf

- Kowalski ML, Ansotegui I, Aberer W, Al-Ahmad M, Akdis M, Ballmer-Weber BK, et al. Risk and safety requirements for diagnostic and therapeutic procedures in allergology: World Allergy Organization Statement. World Allergy Organ J. 2016; 12;9:33.

- Legislation of the Madrid regional government (in Spanish): Legislative order of February 11, 1986, of the Board of Health and Social Welfare, implementing Royal Decree 146/1985, of December 12, on health centers, services, and facilities, which, in Appendix I, Section 2.9, provides a definition of day hospitals.

- Legislation of the Madrid regional government (in Spanish): Legislative order of February 11, 1986, of the Board of Health and Social Welfare, implementing Royal Decree 146/1985, of December 12, on health centers, services, and facilities, which, in Appendix II, Section 3.18, covers the concept of outpatient units.

- Spanish Ministry of Health. Estándares de calidad de cuidados para la seguridad del paciente en los hospitales del SNS Sistema Nacional de Salud. Proyecto SENECA. Informe Técnico 2008. Available at: http://www.msssi.gob.es/organizacion/ sns/planCalidadSNS/docs/SENECA.pdf

- Spanish Ministry of Health. Estrategia de seguridad del paciente del Sistema Nacional de Salud. Periodo 2015-2020. Available at: https://www.seguridaddelpaciente.es/resources/ documentos/2015/Estrategia\%20Seguridad\%20del\%20 Paciente\%202015-2020.pdf

- Spanish Ministry of Health and Consumer Affairs. Plan de Calidad para el Sistema Nacional de Salud. Available at: http:// www.msc.es/organizacion/sns/planCalidadSNS/home.htm
- Spanish Ministry of Health and Consumer Affairs. Validación de indicadores de calidad utilizados en el contexto internacional: indicadores de seguridad de pacientes e indicadores de hospitalización evitable. Madrid: Ministry of Health and Consumer Affairs; 2008

- Ministry of Health and Social Policy. Unidad de Hospital de Día. Estándares y Recomendaciones. Informes, estudios e investigación; 2009 Available at: http:/wwww.msc.es/ organizacion/sns/planCalidadSNS/docs/UnidadHospitalDia. pdf

- Spanish Ministry of Health, Social Services and Equality. Compromiso por la calidad de las sociedades científicas de España. Available at: http://www.msssi.gob.es/organizacion/ sns/planCalidadSNS/cal_sscc.htm

- Novak-Wegrzyn A, Assàad AH, Bahna SL, Bock SA, Sicherer SH, Teuber SS. Work group report: oral food challenge testing. J Allergy Clin Immunol 2009;123:S365-83.

- Olaguíbel Rivera JM. Plan Estratégico de la Sociedad Española de Alergología e Inmunología Clínica (SEAIC) 2011-2018. Retrieved from: http://www.seaic.org/wp-content/plugins/ download-monitor/download. php?id=Plan-EstrategicoSEAIC-21x28.pdf

- Organización Panamericana de la Salud. Política y estrategia regionales para la garantía de la calidad de la atención sanitaria, incluyendo la seguridad del paciente. $27^{\mathrm{a}}$ Conferencia Sanitaria Panamericana. 59a Sesión del Comité Regional. Resolución CSP27.r10. Washington, D.C, 2007.

- Terol E, Agra Y, Fernandez-Maillo MM, Casal J, Sierra E, Bandres B, Garcia MJ, del Peso P. The Spanish National Health System patient safety strategy, results for the period 20052007. Med Clin (Barc) 2008;131 Suppl 3:4-11.

- World Health Organization. Patient Safety. World Alliance for Patient Safety. Patient Safety. The Launch of the World Alliance for Patient Safety, Washington DC, USA — 27 October 2004. Available at: http://www.who.int/patientsafety/worldalliance/ en/

- World Health Organization Europe. A brief synopsis on Patient safety. WHO Regional Office for Europe. Copenhagen, 2010. Available at: http://www.euro.who.int/_data/assets/pdf_ file/0015/111507/E93833.pdf

- World Health Organization: From information to action. Reporting and learning for patient safety. Patient safety. Available at: http://www.who.int/patientsafety/ implementation/reporting_and_learning/en/

- World Health Organization. World Alliance for Patient Safety. Patient Safety Solutions, 2007. Available at: http://www.who. int/mediacentre/news/releases/2007/pr22/en/

- Working Group of WHO Patient Safety. Human Factors in Patient Safety Review of Topics and Tools. Report for Methods and Measures. WHO 2009; available at: http://www.who.int/ patientsafety/research/methods_measures/human_factors/ human_factors_review.pdf 
Appendix I. QUALITY MANUAL FOR POLLEN AND FUNGAL-SPORE COLLECTION SYSTEMS.

\section{PHYSICAL SPACE AND MATERIALS}

- VOLUMETRIC SUCTION SAMPLERS:

Samples should be changed at the same time in order to avoid modifications in the duration of the sampling period. A recommendable time to do this is 12:00, as when the system is mounted, sections of strip corresponding to 24-hour periods are used, and each resulting sample represents a 12-hour period in a day (from 12:00 to 23:59) and 12 hours of the following day (from 0:00 to 11:59 the following day).

- Sample preparation.

The laboratory of the aerobiology unit should contain a clean bench with the following accessories and elements required to mount the slides and analyze the samples:

- Sheet of blotting paper.

- Ruler for sample mounting.

- Microscope slides and cover slips.

- Coating substance (vaseline), glycerogelatin with fuchsin, and a sealant.

TIME:

Sample preparation takes 15 to 30 minutes depending on the sample size ( 1 or 7 days).

- Sample analysis. Counting.

The recording period varies greatly and depends on a number of factors. In spring, a 1-day recording may take over 60 minutes, while in summer this may take only 10 minutes. The following equipment is necessary for this procedure:

- Optical microscope for sample reading (400- to $1000 \times$ magnification)

- Oil used for immersion

- Format for results expression

TIME:

It takes about 10 minutes to upload data to the server.

\section{STAFF}

Individuals in charge of taking pollen and fungal-spore readings should hold a university degree or vocational-level training and have accredited experience in aerobiology, having passed the quality-control certification measures for identifying pollen and fungal spores, be skilled in the use of a microscope, and have basic computer skills. 
TECHNIQUES SPECIFIC TO ALLERGY MEDICINE:

- Measurement of total IgE levels

- Measurement of specific IgE levels

- Specific IgE to whole-allergen extracts

- Specific IgE to antigen components

- Tryptase assay

- Eosinophil cationic protein (ECP) measurement

- Preparation of allergen extracts for in vivo diagnosis

- Measurement of basophil activation

\section{PHYSICAL SPACE}

The minimum size for this space will depend on the number of machines. If this unit has an ImmunoCAP 250, an ImmunoCAP ISAC, a flow cytometer, and separate refrigerator and freezer, in addition to a work space and area for the drafting of reports, this space should measure $40 \mathrm{~m}^{2}$. However, if part of this space is shared with other laboratories such as immunology or laboratory testing, $20 \mathrm{~m}^{2} \mathrm{may}$ suffice. For the purposes of this document, we have classified the laboratory as an optional element, as in some facilities the allergy laboratory is housed within the allergy department proper, in which case the aforementioned requirements apply. In most facilities this space is one of the sections of the central laboratory (commonly that of immunology) and is not organizationally attached to the allergy department.

\section{MATERIAL RESOURCES}

- Space for storing samples, equipment, and a work area.

- Equipment necessary for storing samples and reagents that must be refrigerated $\left(4^{\circ} \mathrm{C}\right)$ and others that must be kept frozen $\left(-30^{\circ} \mathrm{C}\right)$.

- Work bench for processing samples, furnished for the purpose of washing.

- Pipettes in which to process small quantities of reagents and samples: p10, p20, p200, and p1000 (or their equivalents) as well as disposable plastic tips for use with these pipettes.

- Incubation bath with shaking function.

- Vortex mixer.

- Centrifuges (for separating sera and cells).

- Flow cytometry.

- InmunoCAP 250 (Thermofisher) or equivalent.

- Microarray technology (ImmunoCAP (Thermofisher) or equivalent).

- Computer systems for reports.

\section{STAFF}

Laboratory and university-trained technician or a specialist in allergy or immunology to validate results and draft reports. 


\section{PROJECT OBJECTIVES}

Provide a set of minimum criteria for the management of allergic disease in allergy departments and units in adherence of utmost quality and safety standards, thereby serving as guidelines to be used by the relevant authorities, suppliers, and care professionals who are stakeholders in these matters.

To arrive at a certain degree of consensus among the allergists involved in this initiative with regard to some of the more controversial recommendations for safety and quality in allergy departments and units appearing in the "Document on Quality and Safety Standards in Allergy Medicine" published by SEAIC, a participatory process was designed in accordance with the Delphi method.

\section{METHODOLOGY}

Process of consensus determination via the Delphi method.

- April 2017: Delphi protocol and survey drafted

- May 2017: Parameter-setting of the web-based platform

- June 2017: 1st round of Delphi method

- July 2017: 2nd round of Delphi method

- September 2017: Report with final results

\section{PARTICIPANT ROLES}

- Organizing Committee (authors of this document)

- Steer and oversee the entire process.

- Name the members of the Scientific Committee.

- Propose experts with sufficient expertise to participate in the survey.

- Scientific Committee (project contributors)

- Take part in deciding on the methodological issues addressed in the project.

- Collaborate in drafting and validating the questionnaire.

- Act as advisers for the analysis and interpretation of results.

- Propose experts with sufficient expertise to participate in the survey.

- Panel of experts

- Complete the survey.

\section{METHODOLOGY}

Consensus criteria

- Unanimity: $100 \%$ of panelists agree with the statement.

- Consensus: When at least $90 \%$ of panelists agree with the statement, though without reaching unanimity.

- Majority: When $\geq 66 \%$ and $<90 \%$ of the expert panel agree.

- Disagreement: When $<66 \%$ of experts surveyed agree with the statement.

237 allergists and nurses $(n=3)$ were invited to take part; the response rate was $66 \%$ (175 responders in the first round). For the second round, the questionnaire was sent to the 175 responders from the previous round, with $153(86 \%)$ responding. 
RESULTS. Global first and second round

\begin{tabular}{|c|c|c|c|c|c|c|c|c|c|}
\hline \multirow[t]{2}{*}{ Firts round } & \multicolumn{2}{|c|}{ Unanimity } & \multicolumn{2}{|c|}{ Consensus } & \multicolumn{2}{|c|}{ Majority } & \multicolumn{2}{|c|}{ Disagreement } & \multirow{2}{*}{$\begin{array}{c}\text { Total } \\
\mathrm{n} \\
\end{array}$} \\
\hline & $\%$ & $\mathrm{n}$ & $\%$ & $\mathrm{n}$ & $\%$ & $\mathrm{n}$ & $\%$ & $\mathrm{n}$ & \\
\hline I. Materials & $0 \%$ & 0 & $55.56 \%$ & 5 & $22.22 \%$ & 2 & $22.22 \%$ & 2 & 9 \\
\hline II. Structure and physical space & $0 \%$ & 0 & $13.79 \%$ & 4 & $58.62 \%$ & 17 & $27.59 \%$ & 8 & 29 \\
\hline III. Staff & $0 \%$ & 0 & $30.00 \%$ & 6 & $30.00 \%$ & 6 & $40.00 \%$ & 8 & 20 \\
\hline \multirow[t]{2}{*}{ IV. Time } & $0 \%$ & 0 & $3.23 \%$ & 1 & $67.74 \%$ & 21 & $29.03 \%$ & 9 & 31 \\
\hline & & & & & & & \multicolumn{3}{|c|}{$\begin{array}{c}\mathrm{N}(\mathrm{I})=273 \rightarrow \mathrm{N}(\mathrm{f})=179 \\
66 \% \text { response }\end{array}$} \\
\hline \multirow[t]{2}{*}{ Second round } & \multicolumn{2}{|c|}{ Unanimity } & \multicolumn{2}{|c|}{ Consensus } & \multicolumn{2}{|c|}{ Majority } & \multicolumn{2}{|c|}{ Disagreement } & Total \\
\hline & $\%$ & $\mathrm{n}$ & $\%$ & $\mathrm{n}$ & $\%$ & $\mathrm{n}$ & $\%$ & $\mathrm{n}$ & $\mathrm{n}$ \\
\hline I. Materials & $0 \%$ & 0 & $100 \%$ & 2 & $0 \%$ & 0 & $0 \%$ & 0 & 2 \\
\hline II. Structure and physical space & $0 \%$ & 0 & $57.14 \%$ & 12 & $38.10 \%$ & 8 & $4.76 \%$ & 1 & 21 \\
\hline III. Staff & $0 \%$ & 0 & $85.71 \%$ & 6 & $14.29 \%$ & 1 & $0 \%$ & 0 & 7 \\
\hline \multirow[t]{2}{*}{ IV. Time } & $0 \%$ & 0 & $43.75 \%$ & 14 & $37.50 \%$ & 12 & $18.75 \%$ & 6 & 32 \\
\hline & & & & & & & \multicolumn{3}{|c|}{$\begin{array}{c}\mathrm{N}(\mathrm{I})=178 \rightarrow \mathrm{N}(\mathrm{f})=153 \\
86 \% \text { response }\end{array}$} \\
\hline \multirow[t]{2}{*}{ Firts and second round } & \multicolumn{2}{|c|}{ Unanimity } & \multicolumn{2}{|c|}{ Consensus } & \multicolumn{2}{|c|}{ Majority } & \multicolumn{2}{|c|}{ Disagreement } & Total \\
\hline & $\%$ & $\mathrm{n}$ & $\%$ & $\mathrm{n}$ & $\%$ & $\mathrm{n}$ & $\%$ & $\mathrm{n}$ & $\mathrm{n}$ \\
\hline I. Materials & $0 \%$ & 0 & $100 \%$ & 7 & $0 \%$ & 0 & $0 \%$ & 0 & 7 \\
\hline II. Structure and physical space & $0 \%$ & 0 & $64 \%$ & 16 & $32 \%$ & 8 & $4 \%$ & 1 & 25 \\
\hline III. Staff & $0 \%$ & 0 & $92.31 \%$ & 12 & $7.69 \%$ & 1 & $0 \%$ & 0 & 13 \\
\hline IV. Time & $0 \%$ & 0 & $45.45 \%$ & 15 & $36.36 \%$ & 12 & $18.18 \%$ & 6 & 33 \\
\hline Total & $0 \%$ & 0 & $75.44 \%$ & 50 & $19.01 \%$ & 21 & $5.55 \%$ & 7 & 78 \\
\hline
\end{tabular}


I. MATERIALS

Challenge tests using physical agents:

- When diagnosing physical (inducible) urticarias, it is recommended to use the specific materials recommended in published protocols, as these allow the procedure to be performed in the most suitable manner $(97.39 \%)$.

Protocols and incident-reporting systems

- Standard operating procedures (SOP), including support for recording data relevant to the procedure while it is ongoing, such as vital signs, dose levels administered, etc. (96.65\%).

- Protocols/standard technical procedures should appear in writing in the event of serious adverse reactions and should be available to all team members $(96.09 \%)$.

- Protocol for treating adverse reactions (with a code for cardiac arrest) (97.21\%).

- System for reporting incidents (94.97\%).

- Staff with training in CPR, achieved by taking a course every 2-3 years $(91.50 \%)$.

- Staff with specific training in CPR (98.32\%).

\section{STRUCTURE AND PHYSICAL SPACE}

Food/drug challenge tests

- A low-risk food challenge is that which has a low risk of causing a reaction and one in which the risk of a severe reaction is low (97.39\%).

- A moderate-risk food challenge is that which has a low or a high risk of causing a reaction and one in which the risk of a severe reaction is low $(83.01 \%)$.

- A high-risk food challenge refers to any test that involves a high risk of a severe reaction (94.97\%).

- A patient at high risk of developing a reaction to a food is considered to be one having a history of allergic reaction to the culprit food over the past 6-12 months or who has unfavorable results in tests for allergens (i.e. skin test or highly positive IgE or sensitization to allergen components that have a strong association to clinical relevance, such as ara $\mathrm{h} 2)(93.46 \%)$.

- It is considered that a patient at low risk of developing a severe reaction to a food is one who has never had a severe reaction, does not have asthma, and in whom the food studied is not usually related to anaphylactic reactions or have a molecular sensitization profile that is linked to severe reactions $(97.39 \%)$.

- Patients believed to be at high risk of a severe allergic reaction to a food are those who have a past history of a severe reaction, or are asthmatic, or have developed a moderate/severe reaction to traces of the food, or who present certain conditions that may hamper the treatment of anaphylaxis, should it occur (e.g. cardiovascular disease, difficult vascular access or intubation, betablocker medication use); additionally, a patient is considered to be at high risk if the food being studied is frequently implicated in severe reactions (e.g. peanut, nuts, fish, seafood, seeds) or is sensitized to allergen components associated with severe reactions $(94.97 \%)$.

- Regardless of the expected severity of the reaction, it is recommended that all provocation challenges with food be performed in an $\mathrm{ADH}$ due to the duration of the test $(73.86 \%)$.

- Moderate-risk food challenges require an $\mathrm{ADH}(90.20 \%)$.

- Challenges with foods that pose a moderate risk require an $\mathrm{ADH}(90.20 \%)$; and this unit should be located on the premises of a hospital (83.01\%).

- High-risk food challenges require an $\mathrm{ADH}$, which should be located within a hospital facility $(95.53 \%)$.

- Desensitization to food allergens and controlled challenge tests have an extremely high level of risk and high complexity, due either to the severity of the reaction or because of existing comorbidities that increase the risk of anaphylaxis. These activities must be performed in an $\mathrm{ADH}$, though potentially with the support of intensive care units $(96.73 \%)$.

- To carry out controlled challenge tests of immediate reactions to drugs, it is recommended to have an ADH hospital independently of the degree of risk $(94.12 \%)$.

- It is recommended to perform controlled challenge tests for non-immediate drug reactions in an ADH facility (73.20\%).

- It is recommended to perform controlled challenge tests for non-immediate drug reactions in an ADH facility (94.97\%).

- Desensitization to drugs and controlled challenge tests have an extremely high level of risk and high complexity, due either to the severity of the reaction or because of existing comorbidities that increase the risk of anaphylaxis. These procedures must be carried out in an ADH located within a facility that has an ICU/postoperative recovery unit/emergency department, where patients who develop a severe reaction may receive treatment $(98.04 \%)$. 


\section{DETAILED RESULTS (continuation)}

- It is recommended that the room where procedures related to challenge tests/desensitization/immunotherapy with foods are carried out and where patients who have undergone the test remain under observation have a minimum floor space of $2 \mathrm{~m}^{2}$ per patient. (Here, floor space per patient refers to the total size of the room, so a $20 \mathrm{~m}^{2}$ space can be used to treat up to 10 patients) (88.24\%).

- An independent space for carrying out intervention-related treatment procedures (food-allergen desensitization/immunotherapy) and monitoring-observation is recommended, though this space may be located in the day hospital, sectioned off for privacy. This should be furnished with a bed or examination table. Each station of this type should measure 4-5 $\mathrm{m}^{2}(92.81 \%)$.

- It inadvisable to perform intraepidermal or intradermal tests on patients with a history of anaphylaxis of grade 2 or higher in specialist clinics located outside of a hospital facility when these lack access to an ICU/postoperative recovery unit/emergency department in under 15-30 minutes $(83.66 \%)$.

Respiratory function tests

- Specific bronchial challenges with allergens do not need to be conducted in the room used for lung-function testing (46.41\%).

Sting challenge

- It is recommended that sting challenges be performed in the ADH provided there is access to an ICU (93.46\%).

- It is recommended to treat any allergic reactions that may occur during a procedure in a room specially designated for treating these reactions $(98.69 \%)$.

- It is recommended that the room for treatment of allergic reactions be adjacent to the room where procedures are performed and feature a system to block visibility of this space by the other patients in the ADH $(94.77 \%)$.

- The minimum recommended size of an ADH reaction-treatment room is $4 \mathrm{~m}^{2}(92.81 \%)$.

- The room for treatment of allergic reactions in the ADH should have 1 bed for every 10 stations devoted to diagnostic and treatment procedures $(85.62 \%)$.

Other procedures

- Specific nasal challenges do not need to be carried out in an $\mathrm{ADH}(79.08 \%)$.

\section{STAFF}

Food/drug challenge tests

- For all controlled challenge tests, it is recommended that there be an allergist either physically present or nearby (98.88\%).

- The presence of nursing staff is recommended for all controlled challenge tests $(94.97 \%)$.

- It is recommended that skin-test results be verified by an allergist belonging to the allergy department; additionally, this allergist shall be easily located should the need for rapid response arise due to allergic reactions taking place during the test procedures $(90.85 \%)$.

- It is recommended to have access to orderlies who, when called, can respond rapidly for rush transfer of patients (92.18\%).

- Members of administrative staff may have responsibilities for both the ADH and the allergy department itself $(86.27 \%)$.

Respiratory function tests

- To perform a spirometry test, it is sufficient to have trained nursing staff physically present and an allergy specialist who can be easily located $(95.53 \%)$.

- To perform a bronchodilator test, it is sufficient to have trained nursing staff physically present and an allergy specialist who can be quickly and easily located $(94.41 \%)$.

Sting challenge

- Controlled sting challenges may be performed by a nurse provided an allergist is present; alternatively, the allergist can carry out the procedure him or herself $(94.12 \%)$.

Other procedures

- Nasal provocation testing may be performed by a trained nurse provided there is an allergist nearby (98.69\%).

- Conjunctival provocation testing with allergen extracts may be performed by a trained nurse provided there is an allergist nearby $(92.74 \%)$.

- Olfactometry tests can be carried out by a trained nurse $(96.73 \%)$.

- Allergy units are recommended to have support from an assistant nurse $(90.85 \%)$.

- It is recommended that there be an allergologist physically present and whose sole responsibility is to oversee the ADH, performing between 10 and 15 procedures per day $(96.08 \%)$. 


\section{DETAILED RESULTS (continuation)}

\section{TIME}

Drug and food challenge tests

- When establishing the time it takes for a nurse to perform a task, not only the times involved to complete the procedure should be factored in, but also the time devoted to preparation, observation, resolution of complications, education, and data recording $(98.88 \%)$.

- On average, nurses should be allotted 15-20 minutes per patient to prepare, perform, and record the results of prick/intradermal skin tests $(69.93 \%)$.

- At least 45 minutes of work by a nurse per patient shall be allotted for skin tests using groups of more complex drugs due to the greater number of agents tested and dilutions used (e.g. general anesthetic drugs, beta-lactam antibiotics, contrast agents for radiological tests) $(93.46 \%)$.

Low-risk challenge tests

- On average, allergists spend 15 minutes per patient on these $(86.93 \%)$.

- Nursing staff devote 15 minutes to this test per patient $(81.70 \%)$.

Moderate-risk challenge tests

- On average, allergists spend 30 minutes per patient on these $(40.52 \%)$.

- Allergists devote an average of 30-45 minutes per patient $(53.59 \%)$

- On average, allergists spend 45 minutes per patient (56.86\%).

- Nursing staff devote an average of 30-45 minutes per patient (46.41\%).

- Nurses devote an average of 45 minutes per patient (52.29\%).

- Nursing staff devote an average of 45-60 minutes per patient (58.82\%).

High-risk tests

- Allergists devote an average of 45-60 minutes per patient (90.20\%).

- Nurses spend an average of 60 minutes per patient (90.85\%).

Observation following challenge tests

- At minimum, patients require an additional 2 hours of observation time after the provocation test has concluded (94.77\%).

Immunotherapy with foods

- Each nurse assigned to oral desensitization procedures should devote no less than 90 minutes per patient (88.89\%).

- For high-risk or high-complexity parenteral desensitization procedures, each member of nursing staff must devote 120 minutes of their time per patient $(92.16 \%)$.

Challenge tests using physical agents

- Tests for diagnosing dermographism/urticaria factitia require 10 minutes of time from nursing staff per patient $(86.93 \%)$.

- Tests for diagnosing heat-induced urticaria require 10 minutes of time from nursing staff per patient $(84.97 \%)$.

- Tests for diagnosing pressure urticaria require an average of 70 seconds of time from nursing staff when a dermographometer is used and 5 minutes per patient when weights or cylinders are used $(82.35 \%)$.

- To conduct phototesting/protoprovocation for solar urticaria, the average time required of nursing staff is 30-60 minutes per patient; this is the same time commitment required of patients undergoing the procedure $(91.50 \%)$.

- The average time required for the test to diagnose cholinergic urticaria is 30 minutes per patient $(91.50 \%)$.

Respiratory function tests

- When performing respiratory-function tests in children, an additional $15 \%$ time period should be budgeted with respect to the time required for adult patients $(97.39 \%)$.

- To screen for nonspecific bronchial hyperresponsiveness (methacholine or adenosine) with a dosimeter, it is recommended to allot 45 minutes per patient $(90.20 \%)$.

- To screen for nonspecific bronchial hyperresponsiveness (methacholine or adenosine) using the tidal volume method, 60 minutes per patient is the recommended time allotment $(94.12 \%)$. 
DETAILED RESULTS (continuation)

Sting challenge

- 5 is the maximum recommended number of sting challenges per day performed by an allergist and a nurse $(83.01 \%)$.

- A fair estimate of the total time required for a sting challenge is 150 minutes $(91.50 \%)$.

- It is recommendable to keep in mind that allergists devote a total of 70 minutes per patient to sting challenges $(75.82 \%)$.

- It is advisable to keep in mind that a sting challenge requires roughly 85 minutes of a nurse's time per patient (78.43\%).

Other procedures

- In terms of physician time required, nasal endoscopy is considered a low-complexity procedure $(<15$ minutes per patient $)$ (78.43\%).

Appointments with allergists

- It is recommended that an initial allergy visit have an average length of 30 minutes (96.08\%).

- It is recommended that all-in-one, high-resolutionallergy appointments have an average length of 45 minutes (94.12\%).

- It is recommended that follow-up allergy appointments have an average length of 15 minutes (90.20\%).

- It is recommended that allergy appointments to present and discuss results have an average length of 15 minutes (89.54\%). 
Table. Panelists participating in the consensus exercise via the Delphi method

\begin{tabular}{|c|c|c|}
\hline Researcher name & Center of employment & Province \\
\hline Miguel Torrecillas Toro & Complejo Hospitalario Universitario de Albacete & Albacete \\
\hline Patricia Prieto Montaño & Complejo Hospitalario Universitario de Albacete & Albacete \\
\hline Mónica Antón Gironés & Hospital Universitario Del Vinalopó & Alicante \\
\hline Carmen María Andreu Balaguer & Hospital Vega Baja & Alicante \\
\hline José Luis García Abujeta & Hospital Marina Baixa & Alicante \\
\hline Carlos Hernando de Larramendi Martínez & Hospital Marina Baixa & Alicante \\
\hline Víctor Soriano Gomis & Hospital General Universitario de Alicante & Alicante \\
\hline Sergia Cruz Granados & Complejo Hospitalario Torrecárdenas & Almeria \\
\hline María Dolores Quiñones Estévez & Hospital Universitario Central de Asturias & Asturias \\
\hline Irán Sánchez Ramos & Dermatology and allergy clinic & Badajoz \\
\hline María Ángeles Gonzalo Garijo & Hospital Universitario Infanta Cristina & Badajoz \\
\hline Antonio Valero Santiago & Hospital Universitario Clinic Barcelona & Barcelona \\
\hline Anna Sala Cunill & Hospital Vall d'Hebron & Barcelona \\
\hline Gustavo Perdomo Gutiérrez & Allercen Clínica de Alergia e Inmunología Clínica & Barcelona \\
\hline Mar Guilarte & Hospital Vall d'Hebron & Barcelona \\
\hline Albert Roger Reig & Hospital Germans Trias i Pujol & Barcelona \\
\hline Irina Diana Bobolea & Hospital Universitario Clinic Barcelona & Barcelona \\
\hline María Mercedes Guillén Biscarri & Hospital Vall d'Hebron & Barcelona \\
\hline Teresa Dordal Culla & Hospital Municipal de Badalona & Barcelona \\
\hline Teresa Garriga Baraut & Hospital Vall d'Hebron & Barcelona \\
\hline Moisés Labrador Horrillo & Hospital Vall d'Hebron & Barcelona \\
\hline Pedro Carretero Anibarro & Hospital Universitario de Burgos & Burgos \\
\hline Maria Isabel Alvarado Izquierdo & Complejo Hospitalario Universitario de Cáceres & Caceres \\
\hline Manuela Alvarado Arenas & Complejo Hospitalario Universitario de Cáceres & Caceres \\
\hline Carmen Domínguez Noche & Hospital Virgen Del Puerto & Caceres \\
\hline Francisco Javier Hernández Arbeiza & Complejo Hospitalario Universitario de Cáceres & Caceres \\
\hline Sergio Luis Porcel Carreño & Hospitalario Nuestra Señora de la Montaña & Caceres \\
\hline Ignacio García Núñez & Hospital Quirónsalud Campo de Gibraltar & Cadiz \\
\hline Francisco Moreno & Private practice & Cadiz \\
\hline Diego Gutiérrez Fernandez & Hospital Universitario Puerta del Mar & Cadiz \\
\hline Francisco David El-Qutob López & Hospital Universitario de la Plana & Castellon \\
\hline Rosa García Rodríguez & Hospital General Universitario de Ciudad Real & Ciudad Real \\
\hline Luis Alonso González Sánchez & Complejo Hospitalario La Mancha Centro & Ciudad Real \\
\hline Teresa Alfaya Arias & Hospital General Universitario de Ciudad Real & Ciudad Real \\
\hline Ana María Burgos Montero & Complejo Hospitalario La Mancha Centro & Ciudad Real \\
\hline Pilar Mur Gimeno & Hospital Santa Bárbara & Ciudad Real \\
\hline Francisco Feo Brito & Hospital General Universitario de Ciudad Real & Ciudad Real \\
\hline Carmen Moreno Aguilar & Hospital Universitario Reina Sofía & Cordoba \\
\hline Berta Ruiz Leon & Hospital Universitario Reina Sofía & Cordoba \\
\hline Fernando Florido López & Hospital Universitario San Cecilio & Granada \\
\hline Arantza Vega Castro & Hospital Universitario de Guadalajara & Guadalajara \\
\hline Juan María Beitia Mazuecos & Hospital Universitario de Guadalajara & Guadalajara \\
\hline
\end{tabular}


Table. Panelists participating in the consensus exercise via the Delphi method (continuation)

\section{Researcher name}

Remedios Cárdenas Contreras

Alejandro Joral Badas

María Ascensión Aranzábal Soto

Jose Antonio Navarro Echeverria

Maria Cesárea Sánchez Hernández

Blanca Sáenz de San Pedro Morera

Antonio Parra Arrondo

Pilar Iriarte Sotés

Virginia Rodríguez Vázquez

Carmen Vidal Pan

Teófilo Lobera Labairu

Nancy R. Ortega Rodríguez

Beatriz Fernandez Parra

Lluis Marques Amat

Joaquín Martín Lázaro

Sonsoles Infante Herrero

Miguel Angel Tejedor

Sandra Blanco Bermejo

Teresa Valbuena

Jose Julio Laguna Martinez

Jose Manuel Zubeldia Ortuño

Francisco Javier Contreras Porta

Montserrat Fernandez Rivas

Joaquín Sastre Domínguez

Sonia Vázquez

María Teresa Caballero Molina

Beatriz Huertas Barbudo

Tomás Chivato Pérez

Silvia Sanchez Garcia

Javier Domínguez Ortega

Mercedes Rodriguez Rodriguez

María Victoria Múgica Garcia

Lys Herráez

Margarita Tomas Perez

Ana Rosado Ingelmo

Matilde Rodriguez Mosquera

Maria Luisa Gonzalez Gutiérrez

Rosario González Mendiola

Luis Manso Alonso

David González de Olano

Belén de la Hoz Caballer

María Cristina Mañas Rueda

\section{Center of employment}

Hospital Universitario de Guadalajara

Hospital Universitario Donostia

Hospital de Zumárraga

Hospital Universitario Donostia

Hospital Juan Ramón Jimenez

Complejo Hospitalario de Jaén

Hospital Universitario A Coruña

Complejo Hospitalario Universitario de Ferrol

X.X.I. de Santiago

Hospital Clínico Universitario de Santiago

Hospital San Pedro

Hospital Universitario de Gran Canaria Doctor Negrín

Hospital del Bierzo

Hospital Universitari Arnau de Vilanova

Hospital Universitario Lucus Augusti de Lugo

Hospital General Universitario Gregorio Marañón

Hospital de Alcorcón

Hospital Infanta Elena (Valdemoro)

Hospital Infanta Sofía

Hospital Central Cruz Roja

Hospital General Universitario Gregorio Marañón

Hospital Universitario La Paz

Hospital Clínico San Carlos

Fundación Jiménez Díaz

Hospital Clínico San Carlos

Hospital Universitario La Paz

Hospital Universitario del Sureste

Private clinic

Hospital Infantil Universitario Niño Jesús

Hospital Universitario La Paz

Hospital Universitario Príncipe de Asturias

Hospital Universitario de La Princesa

Hospital 12 de Octubre

Hospital Universitario La Paz

Hospital Universitario Fundación Alcorcón

Hospital Universitario Puerta de Hierro-Majadahonda

Hospital Universitario Clínico de San Carlos

Hospital Central Cruz Roja

Hospital Universitario del Sureste

Hospital Universitario Ramón y Cajal

Hospital Universitario Ramón y Cajal

Hospital Universitario La Paz
Province

Guadalajara

Guipuzcoa

Guipuzcoa

Guipuzcoa

Huelva

Jaen

La Coruña

La Coruña

La Coruña

La Coruña

La Rioja

Las Palmas

Leon

Lleida

Lugo

Madrid

Madrid

Madrid

Madrid

Madrid

Madrid

Madrid

Madrid

Madrid

Madrid

Madrid

Madrid

Madrid

Madrid

Madrid

Madrid

Madrid

Madrid

Madrid

Madrid

Madrid

Madrid

Madrid

Madrid

Madrid

Madrid

Madrid 
Table. Panelists participating in the consensus exercise via the Delphi method (continuation)

\begin{tabular}{|c|c|c|}
\hline Researcher name & Center of employment & Province \\
\hline Valentín López Carrasco & Hospital Universitario La Paz & Madrid \\
\hline Pedro Ojeda Fernandez & Clínica Ojeda de Asma y Alergia & Madrid \\
\hline Santiago Quirce Gancedo & Hospital Universitario La Paz & Madrid \\
\hline María del Mar Fernández Nieto & Fundación Jiménez Díaz & Madrid \\
\hline Mar Moro & Hospital Universitario Fundación Alcorcón & Madrid \\
\hline Carmelo Escudero Díez & Hospital Infantil Universitario Niño Jesús & Madrid \\
\hline Alicia Prieto García & Hospital General Universitario Gregorio Marañón & Madrid \\
\hline María Teresa Belver González & Hospital Universitario de La Princesa & Madrid \\
\hline Beatriz Núñez Acevedo & Hospital Universitario Infanta Sofía & Madrid \\
\hline Pablo Rodríguez del Río & Hospital Infantil Universitario Niño Jesús & Madrid \\
\hline Francisco Vega de la Osada & Hospital Universitario de La Princesa & Madrid \\
\hline Maria Magdalena Lluch Bernal & Hospital Universitario La Paz & Madrid \\
\hline Eloina Gonzalez Mancebo & Hospital Universitario de Fuenlabrada & Madrid \\
\hline Antonio José Pérez Pimiento & Hospital Universitario Puerta de Hierro-Majadahonda & Madrid \\
\hline Alfredo Iglesias Cadarso & Hospital Universitario Puerta de Hierro-Majadahonda & Madrid \\
\hline Darío Antolín Amérigo & Hospital Príncipe de Asturias & Madrid \\
\hline Javier Ruiz Hornillos & Hospital Universitario Infanta Elena & Madrid \\
\hline Leticia Sánchez Morillas & Hospital Universitario Clínico de San Carlos & Madrid \\
\hline Paloma (María Dolores) Ibáñez Sandín & Hospital Infantil Universitario Niño Jesús & Madrid \\
\hline Alberto Álvarez-Perea & Hospital General Universitario Gregorio Marañón & Madrid \\
\hline Mar Reaño Martos & Hospital Universitario Puerta de Hierro-Majadahonda & Madrid \\
\hline Francisco Javier Ruano Perez & Hospital Universitario Infanta Leonor & Madrid \\
\hline Gonzalo Campos Suárez & Private clinic & Malaga \\
\hline María José Torres Jaén & Hospital Regional Universitario de Málaga & Malaga \\
\hline Candelaria Muñoz Román & Hospital Regional Universitario de Málaga & Malaga \\
\hline José María Vega Chicote & Hospital Regional Universitario de Málaga & Malaga \\
\hline José Damián López Sánchez & Hospital Clínico Universitario Virgen de la Arrixaca & Murcia \\
\hline Juan Carlos Miralles López & Hospital General Universitario Reina Sofía & Murcia \\
\hline Ana Isabel Tabar Purroy & Complejo Hospitalario de Navarra & Navarre \\
\hline Blanca E. García Figueroa & Complejo Hospitalario de Navarra & Navarre \\
\hline María J. Álvarez Puebla & Complejo Hospitalario de Navarra & Navarre \\
\hline José María Olaguibel Ribero & Complejo Hospitalario de Navarra & Navarre \\
\hline Jorge Darío Méndez Alcalde & Hospital Río Carrión & Palencia \\
\hline M Jose Garcia Romo & Complejo Asistencial Universitario de Salamanca & Salamanca \\
\hline Milagros Lázaro Sastre & Complejo Asistencial Universitario de Salamanca & Salamanca \\
\hline Esther Moreno Rodilla & Complejo Asistencial Universitario de Salamanca & Salamanca \\
\hline Ignacio Dávila González & Complejo Asistencial Universitario de Salamanca & Salamanca \\
\hline Ana María Navarro Pulido & Hospital El Tomillar & Sevilla \\
\hline Virginia Bellido Linares (private) & Hospital Universitario Virgen Macarena & Sevilla \\
\hline Cristina López Ruiz & Complejo Hospitalario Huelva & Sevilla \\
\hline María del Pilar Lara de la Rosa & Hospital Universitario Virgen Macarena & Sevilla \\
\hline Esther Velázquez & Quirón Sagrado Corazón & Sevilla \\
\hline
\end{tabular}




\begin{tabular}{|c|c|c|}
\hline Yolanda Puente Crespo & Hospital Universitario Virgen Macarena & Sevilla \\
\hline Julio Delgado Romero & Hospital Universitario Virgen Macarena & Sevilla \\
\hline Virginia de Luque Piñana & Hospital Universitario Virgen Macarena & Sevilla \\
\hline Paloma Poza Guedes & Hospital Universitario de Canarias & $\begin{array}{l}\text { Santa Cruz } \\
\text { de Tenerife }\end{array}$ \\
\hline Ruperto Gonzalez Perez & Hospital Universitario de Canarias & $\begin{array}{l}\text { Santa Cruz } \\
\text { de Tenerife }\end{array}$ \\
\hline Francisco Javier Iglesias Souto & Hospital Universitario Nuestra Señora de Candelaria & $\begin{array}{l}\text { Santa Cruz } \\
\text { de Tenerife }\end{array}$ \\
\hline Pere Gaig & Hospital Universitari Joan XXIII & Tarragona \\
\hline Maria del Mar Jimenez Lara & Hospital Virgen del Valle & Toledo \\
\hline Álvaro Moreno Ancillo & Hospital Nuestra Señora del Prado de Talavera & Toledo \\
\hline Nieves Cabañes Higuero & Hospital Virgen del Valle & Toledo \\
\hline Carlos Senent Sanchez & Hospital Virgen del Valle & Toledo \\
\hline Isabel María Sánchez Matas & Hospital Virgen del Valle & Toledo \\
\hline Ángel Moral de Gregorio & Hospital Virgen del Valle & Toledo \\
\hline Dolores Hernández F. de Rojas & Hospital La Fe & Valencia \\
\hline Carmen Pérez Francés & Hospital Universitario Dr. Peset & Valencia \\
\hline M. Jose Pla Martí & Hospital Universitario de la Ribera & Valencia \\
\hline Luis Ángel Navarro Seisdedos & Hospital Público Lluis Alcanyis & Valencia \\
\hline Javier Montoro Lacomba & Hospital Arnau de Vilanova & Valencia \\
\hline María Eugenia Sanchís Merino & Hospital Universitario Rio Hortega & Valladolid \\
\hline Ana M. Alonso Llamazares & Hospital Universitario de Basurto & Vizcaya \\
\hline Ignacio Jáuregui Presa & Hospital Universitario de Basurto & Vizcaya \\
\hline Ignacio Antepara & Hospital Universitario de Basurto & Vizcaya \\
\hline Mar Garcés Sotillos & Hospital Clínico Universitario Lozano Blesa & Zaragoza \\
\hline Jesús Pola Pola & Policlínica Cruz Roja & Zaragoza \\
\hline José Luis Cubero Saldaña & Hospital Clínico Universitario Lozano Blesa & Zaragoza \\
\hline Juan Fraj Lázaro & Hospital Clínico Universitario Lozano Blesa & Zaragoza \\
\hline Ignacio Pérez Camo & Hospital Royo Villanova & Zaragoza \\
\hline
\end{tabular}

